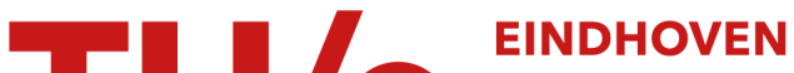 \\ UNIVERSITY OF \\ TECHNOLOGY
}

\section{Robust partial synchronization of delay-coupled networks}

Citation for published version (APA):

Su, L., Wei, Y., Michiels, W., Steur, E., \& Nijmeijer, H. (2020). Robust partial synchronization of delay-coupled networks. Chaos, 30(1), [013126]. https://doi.org/10.1063/1.5111745

\section{Document license:}

TAVERNE

DOI:

10.1063/1.5111745

Document status and date:

Published: 16/01/2020

\section{Document Version:}

Publisher's PDF, also known as Version of Record (includes final page, issue and volume numbers)

\section{Please check the document version of this publication:}

- A submitted manuscript is the version of the article upon submission and before peer-review. There can be important differences between the submitted version and the official published version of record. People interested in the research are advised to contact the author for the final version of the publication, or visit the $\mathrm{DOI}$ to the publisher's website.

- The final author version and the galley proof are versions of the publication after peer review.

- The final published version features the final layout of the paper including the volume, issue and page numbers.

Link to publication

\section{General rights}

Copyright and moral rights for the publications made accessible in the public portal are retained by the authors and/or other copyright owners and it is a condition of accessing publications that users recognise and abide by the legal requirements associated with these rights.

- Users may download and print one copy of any publication from the public portal for the purpose of private study or research.

- You may not further distribute the material or use it for any profit-making activity or commercial gain

- You may freely distribute the URL identifying the publication in the public portal.

If the publication is distributed under the terms of Article $25 \mathrm{fa}$ of the Dutch Copyright Act, indicated by the "Taverne" license above, please follow below link for the End User Agreement:

www.tue.nl/taverne

\section{Take down policy}

If you believe that this document breaches copyright please contact us at:

openaccess@tue.nl

providing details and we will investigate your claim. 


\section{Robust partial synchronization of delay- coupled networks ${ }^{\circ}$}

Cite as: Chaos 30, 013126 (2020); https://doi.org/10.1063/1.5111745

Submitted: 29 May 2019 . Accepted: 09 December 2019 . Published Online: 16 January 2020

Libo Su (D), Yanling Wei, Wim Michiels (D), Erik Steur, and Henk Nijmeijer

\section{COLLECTIONS}

EP This paper was selected as an Editor's Pick
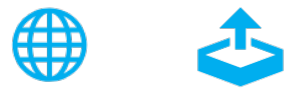

\section{ARTICLES YOU MAY BE INTERESTED IN}

Characterizing the complexity of time series networks of dynamical systems: A simplicial approach

Chaos: An Interdisciplinary Journal of Nonlinear Science 30, 013109 (2020); https:// doi.org/10.1063/1.5100362

How entropic regression beats the outliers problem in nonlinear system identification Chaos: An Interdisciplinary Journal of Nonlinear Science 30, 013107 (2020); https:// doi.org/10.1063/1.5133386

Reconstructing bifurcation diagrams only from time-series data generated by electronic circuits in discrete-time dynamical systems

Chaos: An Interdisciplinary Journal of Nonlinear Science 30, 013128 (2020); https:// doi.org/10.1063/1.5119187

\section{Scilight}




\title{
Robust partial synchronization of delay-coupled networks 타
}

\author{
Cite as: Chaos 30, 013126 (2019); doi: 10.1063/1.5111745 \\ Submitted: 29 May 2019 . Accepted: 9 December 2019. \\ Published Online: 16 January 2020

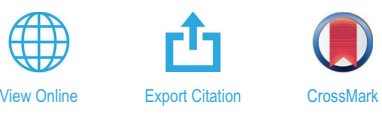

Libo Su, ${ }^{1,2}$ (D) Yanling Wei, ${ }^{7}$ Wim Michiels, ${ }^{1}$ (D) Erik Steur, ${ }^{2,3}$ and Henk Nijmeijer ${ }^{2}$

\author{
AFFILIATIONS \\ ${ }^{1}$ Department of Computer Science, KU Leuven, Celestijnenlaan 200A, 3001 Heverlee, Belgium \\ ${ }^{2}$ Department of Mechanical Engineering, Eindhoven University of Technology, P.O. Box 513, 5600 MB Eindhoven, \\ The Netherlands \\ ${ }^{3}$ Delft Center for Systems and Control, Delft University of Technology, Mekelweg 2, 2628 CD Delft, The Netherlands
}

\begin{abstract}
Networks of coupled systems may exhibit a form of incomplete synchronization called partial synchronization or cluster synchronization, which refers to the situation where only some, but not all, systems exhibit synchronous behavior. Moreover, due to perturbations or uncertainties in the network, exact partial synchronization in the sense that the states of the systems within each cluster become identical, cannot be achieved. Instead, an approximate synchronization may be observed, where the states of the systems within each cluster converge up to some bound, and this bound tends to zero if (the size of) the perturbations tends to zero. In order to derive sufficient conditions for this robustified notion of synchronization, which we refer to as practical partial synchronization, first, we separate the synchronization error dynamics from the network dynamics and interpret them in terms of a nonautonomous system of delay differential equations with a bounded additive perturbation. Second, by assessing the practical stability of this error system, conditions for practical partial synchronization are derived and formulated in terms of linear matrix inequalities. In addition, an explicit relation between the size of perturbation and the bound of the synchronization error is provided.
\end{abstract}

Published under license by AIP Publishing. https://doi.org/10.1063/1.5111745

Synchronization is a form of collective behavior in networks of coupled dynamical systems that plays an important role in a variety of natural and man-made systems. In some networks, partial synchronization-cluster synchronization-may be observed, that is, the network can be divided into clusters such that synchrony of the dynamical systems is only observed within each cluster. In this paper, we derive sufficient conditions for the presence of a relaxed form of partial synchronization in delay-coupled networks, where within a cluster, synchrony only needs to be reached up to some bound. This allows us to take into account the effect of modeling errors and perturbations on the systems and network, which creates more heterogeneity and renders (exact) partial synchronization impossible.

\section{INTRODUCTION}

In the recent decades, the synchronization of networks of interacting dynamical systems has attracted intensive attention. This phenomenon has been widely observed in various fields, ranging from nature to engineering. The unambiguous form of synchronization is full synchronization, which refers to the phenomenon where all the systems behave identically. However, networks may also show a form of incomplete synchronization, called partial synchronization or cluster synchronization, which refers to the phenomenon where some but not all systems behave identically. Partial synchronization often occurs in large, complex systems. One typical example concerns the synchronous activities of neurons in parts of the human brain (see, e.g., Gray, 1994). Another example involves the so-called chimera state, which describes the coexistence of coherence and incoherence in systems of coupled oscillators, introduced in Abrams and Strogatz (2004). Chimera states have been seen in a variety of networked systems such as chemical oscillators (Kuramoto, 1984 and Tinsley et al., 2012), electronic circuits (Schmidt et al., 2014), mechanical oscillators (Martens et al., 2013), optical experiments (Hagerstrom et al., 2012), neural networks (Sawicki et al., 2019), etc.

Sometimes, there may exist time-delays in and between the systems, for instance, due to the transmission time of signals among the systems, which may induce partial synchronization. Several papers have been devoted to studying partial synchronization of networked systems with delayed coupling (see, e.g., Dahms et al., 2012, Orosz, 2014, Steur et al., 2016, and Ryono and Oguchi, 2015). 
From a mathematical point of view, a prerequisite for partial synchronization is the existence of the so-called partial synchronization manifolds, which are positively invariant sets (given a dynamical system, a subset of its state space is called a positively invariant set if it satisfies the property that when the system's state is inside this set at time $t_{0}$, the state will remain within this set for all $t>t_{0}$ ) in the state space of the delay-coupled systems, characterized by the partially synchronized motion. In addition to its existence, the stability of such a manifold is also required for partial synchronization, which guarantees the convergence of whole network states to it (Steur et al., 2012). These concepts are illustrated in Fig. 1. One common method to access the stability of partial synchronization manifold is to analyze an error system describing the deviation between the systems' states within each cluster, which is also called the system of synchronization errors. Therefore, to characterize partial synchronization in delay-coupled systems, two essential steps are needed: (1) identify partial synchronization manifolds and (2) assess the stability of the corresponding synchronization error dynamics given the manifolds. But, for the first step, the conditions used to find partial synchronization manifolds can easily become invalid if a small perturbation is present in the network or the systems.

In Steur et al. (2016) and Su et al. (2018), necessary and sufficient conditions for the existence of partial synchronization manifolds are given. However, the existence conditions in these works are fragile, in the sense that they may be violated by infinitesimal perturbations on coupling weights and delays, as well as perturbations to the dynamical systems at the nodes that create more heterogeneity. As a consequence of such perturbations, the coupled systems may only show an approximate form of synchronization, which means the states (or outputs) of the systems are not completely identical, but remain close to each other within some bound that depends on the size of the perturbation. Inspired of the notion of practical stability, this robust version of synchronization is referred to as practical synchronization. Figure 1 also illustrates an example of such a form of synchronization. As the figure shows the solution may become unable to stay on the manifold because of perturbations. The actual reason behind this is that the system of synchronization errors loses its zero equilibrium due to the perturbations. Without the zero equilibrium, an intuitive expectation is that the errors will stay close to zero if the perturbation is small. However, this is not always the case, for example, when the system is in the vicinity of a perturbation point. Hence, it is important to develop methods to guarantee practical partial synchronization. In addition, due to network imperfections, modeling error, etc., perturbations are often present in the network of coupled systems, thus marking it important to study this type of synchronization. One application of practical synchronization has been shown in Steur et al. (2015), where practical synchronization is used to improve the performance of a neuronal network based controller for driving a mobile robot in an unknown environment while avoiding obstacles.

Several studies have been performed to investigate practical synchronization in delay-free coupled systems. In Steur et al. (2015) and its supplementary material (Steur et al., 2016), the theoretical results on practical synchronization of delay-free, diffusively coupled nonidentical systems with application to Hindmarsh-Rose neurons are presented. It is shown that when the coupling strength is

$x_{3}$

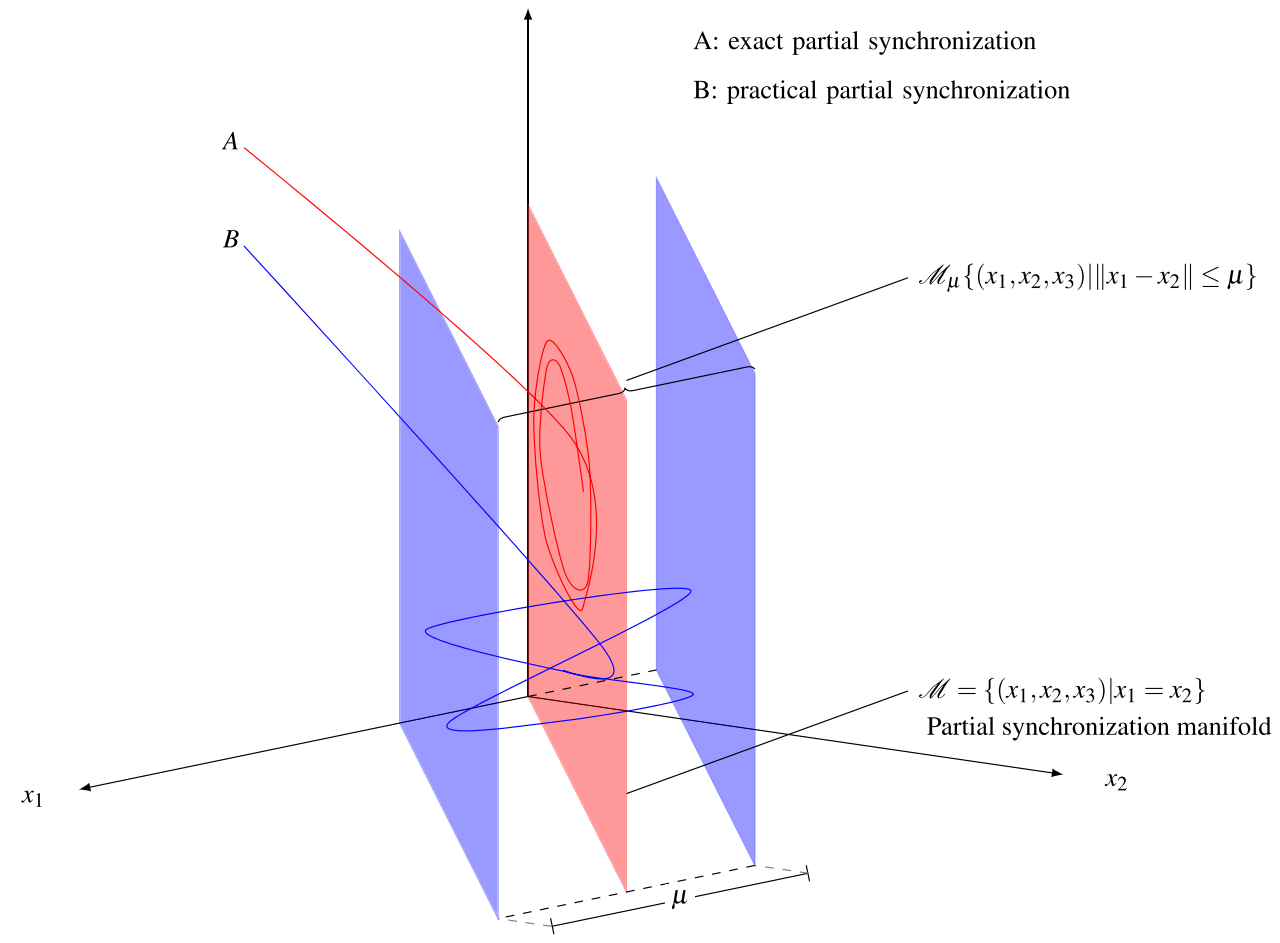

FIG. 1. Exact and practical partial synchronization in a network of three coupled first-order systems. Assume systems 1 and 2 are synchronized. Trajectory A corresponds to the situation where exact partial synchronization occurs. The existence of partial synchronization manifold $\mathscr{M}=\left\{\left(x_{1}, x_{2}, x_{3}\right) \mid x_{1}=x_{2}\right\}$ guarantees that a solution initialized on the manifold (middle plane, in red) stays on this manifold. The stability of the partial synchronization manifold guarantees that neighboring trajectories are attracted to the manifold. Trajectory B corresponds to practical partial synchronization when perturbations are present. In such a case, the trajectory is unable to stay on the middle plane (in red), but it remains inside the space between the two outer planes (in blue), representing the set $\mathscr{M}_{\mu}=\left\{\left(x_{1}, x_{2}, x_{3}\right) \mid\left\|x_{1}-x_{2}\right\| \leq \mu\right\}$. 
sufficiently large, the systems achieve practical synchronization by using the property of semipassivity and convergent system theory. In Panteley and Loria (2017), some preliminary results on practical synchronization of delay-free interconnected heterogeneous oscillators are presented, where the product between the input matrix and output matrix is assumed to be positive definite and the zero dynamics subsystem is convergent. In Montenbruck et al. (2015), by assuming the QUAD property (a system represented by $\dot{x}=f(x, t)$, $f: \mathbb{R}^{n} \times \mathbb{R}^{+} \rightarrow \mathbb{R}^{n}$, is said to be QUAD if $\forall x, y \in \mathbb{R}^{n}$, $(x-y)^{\top}[f(x, t)-f(y, t)] \leq(x-y)^{\top}\left(P-\omega I_{n}\right)(x-y)$, where $P$ is a $n \times n$ diagonal matrix and $\omega$ is a real positive scalar; see DeLellis et al., 2011) for each system, conditions on the interconnection structure of networks of perturbed nonlinear systems with undelayed diffusive couplings are derived to achieve practical synchronization. The benefit of the results in Panteley and Loria (2017), Vromen et al. (2016), Steur et al. (2015), and Montenbruck et al. (2015) is that they provide conceptual insights into the collective behavior of network-interconnected systems with model heterogeneity. However, few work has been done on the quantitative analysis of practical partial synchronization of interconnected systems, in particular, in the presence of delayed couplings.

In this paper, we study the practical partial synchronization of delay-coupled networks. We start with exact partial synchronization as a nominal case. For this, previous results on (exact) partial synchronization in Steur et al. (2016) and Su et al. (2018) are recalled, in particular on the existence of (exact) partial synchronization manifolds. Subsequently, we focus on the case where there are perturbations on the coupling. It is shown that when the perturbations are present, exact partial synchronization becomes, in general, impossible. More precisely, the perturbations typically cause the loss of zero equilibrium of the synchronization errors dynamics. Without the zero equilibrium, the methods used on most works on synchronization, which require a linearization around the equilibrium are not applicable. Here, by connecting the practical partial synchronization with the practical stability of the synchronization error dynamics, we derive conditions for practical partial synchronization. In this practical stability analysis, an equilibrium of the synchronization error dynamics is not required. To this end, first, the dynamics of synchronization errors are isolated from the network dynamics with inclusion of the perturbation; second, by viewing the synchronization error dynamics as a time-delay system affected by perturbations, conditions for the practical stability of such an error system, corresponding to the conditions for practical partial synchronization of the network, are derived. The conditions are formulated in the form of LMIs, which can be efficiently solved by several numerical tools.

The remainder of this paper is organized as follows. In Sec. II, some definitions and assumptions are presented, which are used in the subsequent sections. In Sec. III, the exact practical synchronization is introduced as a nominal case for practical partial synchronization. In addition, an example is used to explain the relevant concepts. It is also shown that small perturbations on the coupling can make exact synchronization impossible and lead to practical partial synchronization. In Sec. IV, the main problem addressed in this paper is formulated, and in Sec. V, the main results are presented. In Sec. VI, a numerical example is presented. Finally, the conclusions are given in Sec. VII.

\section{PRELIMINARIES AND ASSUMPTIONS}

In this paper, we consider networks of systems interacting via linear diffusive time-delay couplings. For such coupled systems, we adopt the settings from Steur et al. (2016). These networks are represented by a directed graph $\mathscr{G}=(\mathscr{V}, \mathscr{E}, A)$, where

- $\mathscr{V}=\{1,2, \ldots, N\}$ is a finite set of nodes with cardinality $|\mathscr{V}|=N$; - $\mathscr{E} \subset \mathscr{V} \times \mathscr{V}$ is the ordered set of edges, where the edge $(i, j)$ points from node $j$ to node $i$; and

- $A=\left(a_{i j}\right) \in \mathbb{R}^{N \times N}$ is the weighted adjacency matrix, where $a_{i j}>0$ represents the weight of edge $(i, j)$ when $(i, j) \in \mathscr{E}$, and $a_{i j}=0$ when $(i, j) \notin \mathscr{E}$.

Besides, the networks considered are simple and strongly connected. A graph is simple if it contains neither self-loops nor multiple edges, where self-loops are edges connecting a node to itself, and multiple edges are two or more edges connecting a same ordered pair of nodes (Gibbons, 1985). A graph is strongly connected if, for any pair of its nodes $i, j$, there exist a directed path from $i$ to $j$ and a directed path from $j$ to $i$ (Bollobas, 1998).

Every node in the networks hosts a dynamical system of the form

$$
\left\{\begin{array}{l}
\dot{x}_{i}(t)=f_{i}\left(x_{i}(t)\right)+B_{i} u_{i}(t), \\
y_{i}(t)=C_{i} x_{i}(t),
\end{array}\right.
$$

where $i \in \mathscr{V}$ states $x_{i}(t) \in \mathbb{R}^{n}$, sufficiently smooth functions $f_{i}$ : $\mathbb{R}^{n} \rightarrow \mathbb{R}^{n}$, inputs $u_{i}(t) \in \mathbb{R}^{m}$, outputs $y_{i}(t) \in \mathbb{R}^{m}$, input matrices $B_{i} \in \mathbb{R}^{n \times m}$, and output matrices $C_{i} \in \mathbb{R}^{m \times n}, i=1, \ldots, N$. Here, we assume all $C_{i} B_{i}$ are similar to positive definite matrices, which guarantees all the systems are left-invertible (the system input-out maps are injective). Left-invertibility is introduced to make the sufficient condition (presented in Sec. III) for the existence of partial synchronization manifolds necessary. One common choice of the matrices $B_{i}$ and $C_{i}$ is $B_{i}=C_{i}=I$, which corresponds to the case of full state feedback.

Systems (1) interact via the following coupling:

$$
u_{i}(t)=k \sum_{j \in \mathscr{N}_{i}} a_{i j}\left[y_{j}(t-\tau)-y_{i}(t)\right]
$$

where $\mathscr{N}_{i}$ is the neighboring set of node $i$, i.e., $\mathscr{N}_{i}:=\{j \in \mathscr{V} \mid(i, j)$ $\in \mathscr{E}\}$, and $\tau$ and $k$ are, respectively, the time-delay and coupling strength.

The state space of the coupled systems (1) and (2) is denoted by $\mathscr{C}\left([-\tau, 0], \mathbb{R}^{N n}\right)$, which is the space of continuous functions mapping the interval $[-\tau, 0]$ into $\mathbb{R}^{N n}$. The network state is defined as below

$$
x_{t} \in \mathscr{C}\left([-\tau, 0], \mathbb{R}^{N n}\right), \quad x_{t}=x(t+\theta), \theta \in[-\tau, 0] .
$$

Next, we introduce the concept of semipassivity to be used to guarantee the boundedness of solutions of the networked systems. The boundedness is later on used in the derivation of conditions for practical partial synchronization. We would like to add that many systems, especially, physical and biological systems, are semipassive.

Definition 1 (Pogromsky, 1998). System (1) is called strictly semipassive if there exist a continuously differentiable, non-negative storage function $V: \mathbb{R}^{n} \rightarrow \mathbb{R}^{+}$and a scalar function $S: \mathbb{R}^{n} \rightarrow \mathbb{R}$, which is positive outside some ball $\mathscr{B}=\left\{x \in \mathbb{R}^{n} \mid\|x\|<R\right\}$ such that

$$
\dot{V}(x(t)) \leq y^{\top}(t) u(t)-S(x(t)) .
$$


Throughout the paper, we make the following assumption.

Assumption 1. Systems (1) are strictly semipassive with a radially unbounded storage function $V$, i.e., $V(x) \rightarrow \infty$ as $\|x\| \rightarrow \infty$.

It has been shown in Steur and Nijmeijer (2011) that Assumption 1 guarantees all solutions of systems (1) coupled via (2) to be ultimately bounded independently of $k$ and $\tau$. More precisely, it has been pointed out in Steur and Nijmeijer (2011) that under this assumption, there exists a bounded set $\Omega$, positively invariant under the dynamics (1) and (2), which attracts all solutions in finite time.

\section{EXACT PARTIAL SYNCHRONIZATION MANIFOLDS}

In this section, we summarize the results from Steur et al. (2016) and Su et al. (2018) on exact practical partial synchronization, which serve as a nominal case in sections IV-VII. In particular, existence conditions of partial synchronization manifolds are recalled. In addition, an example is presented, which shows these conditions can easily become invalid due to a small perturbation. In such a situation, exact synchronization is no longer possible, and only practical synchronization may occur.

For the coupled systems (1) and (2), a solution $x(t)=$ $\left[x_{1}(t)^{\top} \cdots x_{N}(t)^{\top}\right]^{\top}$ is a partially synchronous solution if there exist $i, j \in \mathscr{V}$ with $i \neq j$ such that

$$
x_{i}(t)=x_{j}(t), \quad \forall t \geq t_{0}-\tau .
$$

When a partially synchronous solution exists, the nodes can be grouped into clusters such that the nodes inside each cluster have equal states. To describe this clustering, the concept of partition is used. A partition of $\mathscr{V}$ is a set of nonempty, disjoint subsets of $\mathscr{V}$ whose union is $\mathscr{V}$. The subsets are called parts of the partition which represent the clusters of the nodes. In what follows the total number of parts is denoted by $\kappa$. A partition $\mathscr{P}$ can also be described by an $N \times N$ permutation matrix $\Pi$ such that nodes $i$ and $j$ belong to the same part of the partition if the $i j$ th entry of $\Pi$ is equal to 1 . Note that $\kappa=\operatorname{dim} \operatorname{ker}\left(I_{N}-\Pi\right)$.

By using matrix $\Pi$, the conditions of form (5) can be expressed as $x_{t} \in \mathscr{M}(\Pi)$ for all $t \geq 0$, where

$$
\begin{aligned}
\mathscr{M}(\Pi):= & \left\{\phi \in \mathscr{C}\left([-\tau, 0], \mathbb{R}^{N n}\right) \mid \phi(\theta)\right. \\
= & \operatorname{col}\left(\phi_{1}(\theta), \ldots, \phi_{N}(\theta)\right), \phi_{i}(\theta) \in \mathbb{R}^{n}, i=1, \ldots, N, \\
& \left.\phi(\theta)=\left(\Pi \otimes I_{n}\right) \phi(\theta), \forall \theta \in[-\tau, 0]\right\}
\end{aligned}
$$

is the set of partially synchronous states induced by the permutation matrix $\Pi$. We are now ready to introduce the concept of the partial synchronization manifold.

Definition 2 (Steur et al., 2016). Set $\mathscr{M}(\Pi)$ with permutation matrix $\Pi$ for which $1<\kappa<N$ is a partial synchronization manifold for the coupled systems (1) and (2), if and only if it is positively invariant under the dynamics (1) and (2).

A partition $\mathscr{P}$ associated with $\Pi$ is called viable if the set $\mathscr{M}(\Pi)$ is a partial synchronization manifold. However, identifying a viable partition can be challenging since a direct verification of the invariance property is difficult. In what follows, by introducing another permutation matrix $R$, algebraic conditions for checking the viability of a partition are provided in Lemma 1 , which can be easily verified.
Given a partition, the nodes can be relabeled by cluster such that the first $\kappa_{1}$ nodes belong to the first cluster, the second $\kappa_{2}$ nodes belong to the second cluster, and so on. Mathematically, this can be done by using another permutation matrix $R$ referred as reordering matrix, which can be determined such that

$$
R^{\top} \Pi R=\left[\begin{array}{cccc}
\Pi_{C}\left(\kappa_{1}\right) & & & 0 \\
& \Pi_{C}\left(\kappa_{2}\right) & & \\
& & \ddots & \\
0 & & & \Pi_{C}\left(\kappa_{\kappa}\right)
\end{array}\right],
$$

where $\Pi_{C}\left(\kappa_{i}\right), i=1, \ldots, \kappa$ are $\kappa_{i} \times \kappa_{i}$-dimensional cyclic permutation matrices, that is,

$$
\Pi_{C}\left(\kappa_{i}\right)=\left[\begin{array}{ccccc}
0 & 0 & \cdots & 0 & 1 \\
1 & 0 & \cdots & 0 & 0 \\
0 & \ddots & \ddots & \vdots & \vdots \\
\vdots & \ddots & \ddots & 0 & 0 \\
0 & \cdots & 0 & 1 & 0
\end{array}\right] \in \mathbb{R}^{\kappa_{i} \times \kappa_{i}}
$$

and $\Pi_{C}(1)=1$.

The reordered adjacency matrix can be constructed as

$$
R^{\top} A R=\left[\begin{array}{cccc}
A_{11} & A_{12} & \cdots & A_{1 \kappa} \\
A_{21} & A_{22} & \cdots & A_{2 \kappa} \\
\vdots & \ddots & \ddots & \vdots \\
A_{\kappa 1} & A_{\kappa 2} & \cdots & A_{\kappa \kappa}
\end{array}\right]
$$

with $A_{i j} \in \mathbb{R}^{\kappa_{i} \times \kappa_{j}}$.

Existence conditions for partial synchronization manifolds are formulated in the lemma below.

Lemma 1 (Su et al., 2018). Given an adjacency matrix $A$ and a permutation matrix $\Pi$ of the same dimension, assume that $C_{i} B_{i}$, $i=1, \ldots, N$ are similar to positive definite matrices, then the following statements are equivalent:

(1) $\mathscr{M}(\Pi)$ is a partial synchronization manifold for (1) and (2) and

(2) all blocks of the reordered adjacency matrix (8) have constant rowsums and, in addition, $\mathscr{F}, \mathscr{B}$, and $\mathscr{C}$ defined by

$$
\mathscr{F}:=\left[\begin{array}{c}
f_{1}(\cdot) \\
f_{2}(\cdot) \\
\vdots \\
f_{N}(\cdot)
\end{array}\right], \quad \mathscr{B}:=\left[\begin{array}{c}
B_{1} \\
B_{2} \\
\vdots \\
B_{N}
\end{array}\right], \quad \mathscr{C}:=\left[\begin{array}{c}
C_{1}^{\top} \\
C_{2}^{\top} \\
\vdots \\
C_{N}^{\top}
\end{array}\right]
$$

satisfy the conditions

$$
\mathscr{F}=\left(\Pi \otimes I_{n}\right) \mathscr{F}, \quad \mathscr{B}=\left(\Pi \otimes I_{n}\right) \mathscr{B}, \quad \mathscr{C}=\left(\Pi \otimes I_{n}\right) \mathscr{C} .
$$

Conditions (9) in Lemma 1 express that all the nodes in the same cluster host systems with the same dynamics. Note that this lemma is an extension of Theorems 3 and 4 in Steur et al. (2016), where only networks of identical systems were considered. 


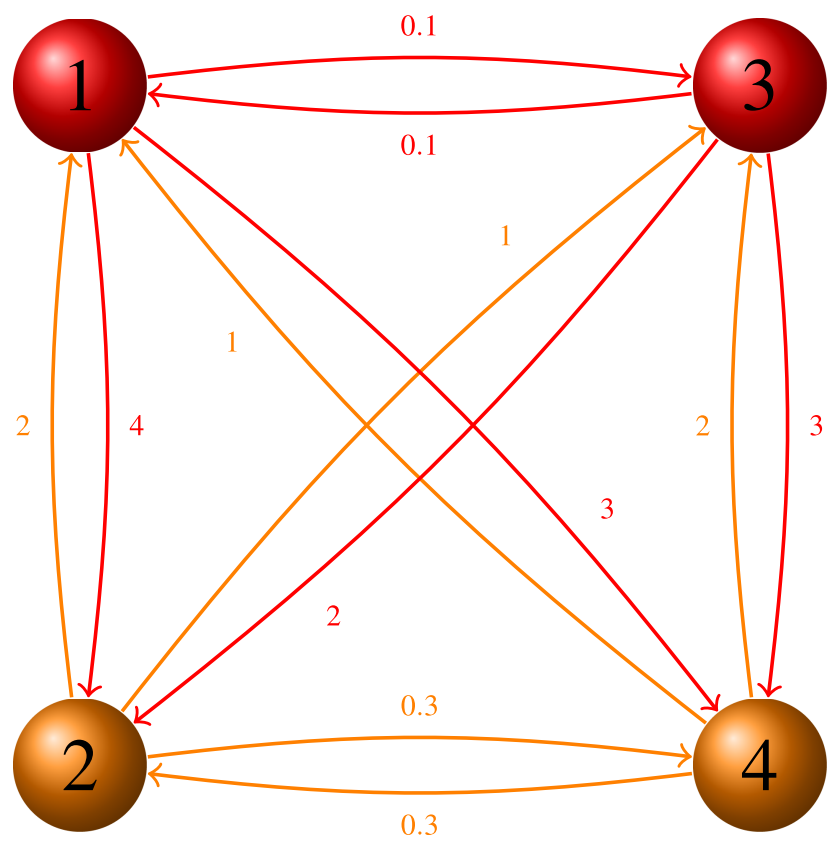

FIG. 2. The network of Example 1 with adjacency matrix $A_{n}$.

Example 1. Consider a network of four nodes shown in Fig. 2. The adjacency matrix of this network is

$$
A_{n}=\left[\begin{array}{cccc}
0 & 2 & 0.1 & 1 \\
4 & 0 & 2 & 0.3 \\
0.1 & 1 & 0 & 2 \\
3 & 0.3 & 3 & 0
\end{array}\right]
$$

We assume that every node hosts a nonlinear system of the following form:

$$
\left\{\begin{array}{l}
\dot{x}_{i, 1}(t)=-\varepsilon x_{i, 1}(t)+x_{i, 2}(t)-x_{i, 1}(t)\left(x_{i, 1}^{2}(t)+x_{i, 2}^{2}(t)\right)+u_{i, 2}(t), \\
\dot{x}_{i, 2}(t)=-x_{i, 1}(t)-\varepsilon x_{i, 2}(t)-x_{i, 2}(t)\left(x_{i, 1}^{2}(t)+x_{i, 2}^{2}(t)\right)+u_{i, 1}(t), \\
y_{i, 1}(t)=x_{i, 2}(t), \\
y_{i, 2}(t)=x_{i, 1}(t), \quad i=1,2,3,4, \varepsilon \in \mathbb{R}
\end{array}\right.
$$

and that the systems are coupled via (2).

By applying Lemma 1 to all possible partitions of this network, we find the only viable partition $\mathscr{P}=\{\{1,3\},\{2,4\}\}$. Software program (available on http://twr.cs.kuleuven.be/research/software/delay -control/manifolds/), based on Lemma 1 , is also available for automatically detecting partial synchronization manifolds. As the network here is relatively simple, the viability of this partition can be easily done by hand. First, since all the systems are identical, conditions (9) are satisfied. Second, the constant row-sum requirement is also met. The corresponding permutation matrix $\Pi$ and the reordering matrix $R$ are given by

$$
\Pi=\left[\begin{array}{llll}
0 & 0 & 1 & 0 \\
0 & 0 & 0 & 1 \\
1 & 0 & 0 & 0 \\
0 & 1 & 0 & 0
\end{array}\right], \quad R=\left[\begin{array}{llll}
1 & 0 & 0 & 0 \\
0 & 0 & 1 & 0 \\
0 & 1 & 0 & 0 \\
0 & 0 & 0 & 1
\end{array}\right] .
$$

The reordered adjacency matrix is

$$
R^{\top} A_{n} R=\left[\begin{array}{cc|cc}
0 & 0.1 & 2 & 1 \\
0.1 & 0 & 1 & 2 \\
\hline 4 & 2 & 0 & 0.3 \\
3 & 3 & 0.3 & 0
\end{array}\right]
$$

Each block in this matrix has constant row-sum.

Remark 1: First, we would like to emphasize that in this example with (10), no full synchronization manifold exists. By definition, a full synchronization manifold is always a subset of any partial synchronization manifold. Therefore, to have partial synchronization, the stability conditions of the partial synchronization manifold should not coincide with the stability conditions of the full synchronization manifold. If no full synchronization manifold exists, this requirement is readily met. Second, from the reordered adjacency matrix, we can see that the coupling of the nodes within clusters is weak and that the coupling of the nodes between clusters is strong (see the ordered adjacency matrix). In fact, this property favors the exact partial synchronization manifold to be stable in the network with $A_{n}$ for some $(k, \tau)$. It might sound counterintuitive but can be explained by the presence of the delay and the sensitivity of high gain feedback with respect to it. Thus, while in the delay-free case high gain coupling favors synchronization, the situation is opposite in the presence of delay (see Su et al., 2018). Without a stable exact synchronization manifold for the nominal network, the practical partial synchronization is unlikely to happen when the network is perturbed. This example network is revisited in Sec. VI for practical partial synchronization study; therefore, we choose this adjacency matrix (10).

To validate the result, a simulation of this network with $k=0.3, \tau=2, \epsilon=-0.2$ has been performed. The results are plotted in Fig. 3. The network is initialized on the partial synchronization manifold, i.e., $x_{1}(\theta)=x_{3}(\theta), x_{2}(\theta)=x_{4}(\theta),-\tau \leq \theta \leq 0$. From Fig. 4, we can see that the systems 1 and 3,2 and 4 remain perfectly synchronized. The states remain on the partial synchronization manifold since they start on the manifold. However, as mentioned before, the existence conditions of such a manifold is fragile. For instance, a small perturbation on the coupling can cause violation of these conditions. Assume there exists a perturbation $\Delta A$ on the adjacency matrix

$$
\begin{aligned}
A=A_{n}+\Delta A= & {\left[\begin{array}{cccc}
0 & 2 & 0.1 & 1 \\
4 & 0 & 2 & 0.3 \\
0.1 & 1 & 0 & 2 \\
3 & 0.3 & 3 & 0
\end{array}\right] } \\
& +\left[\begin{array}{cccc}
0 & 0.2 & 0 & 0 \\
0.2 & 0 & 0 & 0.05 \\
0 & 0 & 0 & 0 \\
0.1 & 0 & 0.2 & 0
\end{array}\right] .
\end{aligned}
$$

With this adjacency matrix, the row conditions in Lemma 1 are not satisfied. The simulation of this perturbed network is shown in 

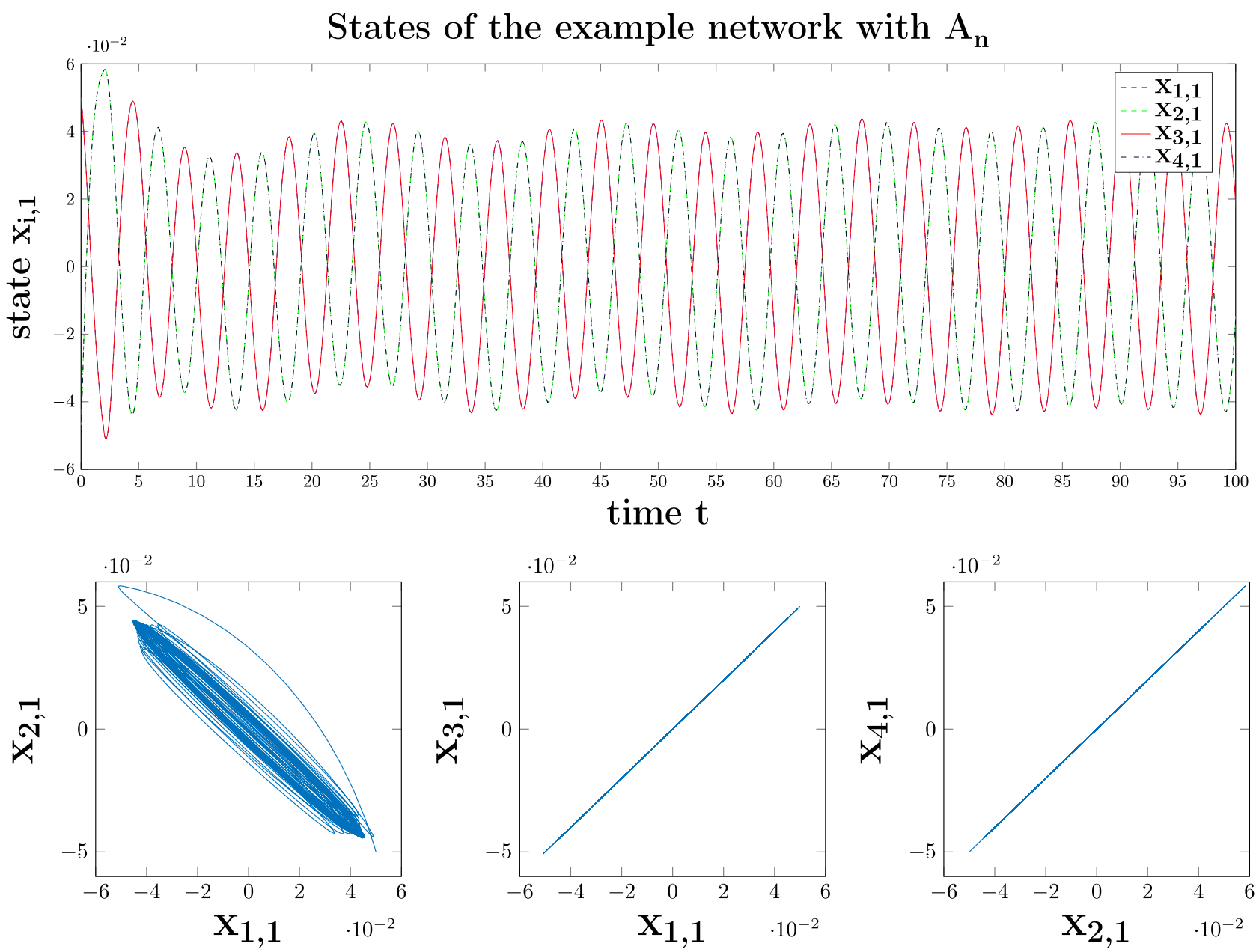

FIG. 3. Simulation of the network corresponding to Example 1, with adjacency matrix $A_{n}$.

Fig. 4. As can be seen from Fig. 4, the sates of systems 1 and 3, 2 and 4 are not identical, but remain close to each other, that is, practical synchronization occurs. This is also confirmed in Fig. 5, which shows the synchronization errors defined as $e_{1}=x_{3,1}-x_{1,1}$, $e_{2}=x_{3,2}-x_{1,2}, e_{3}=x_{4,1}-x_{2,1}$, and $e_{4}=x_{4,2}-x_{2,2}$. In this figure, to better indicate the sizes of these errors, they are expressed as the percentages of maximum magnitudes of the relevant state variables $\left(\frac{e_{1}}{\max _{t}\left|x_{1,1}(t)\right|} \times 100 \%, \frac{e_{2}}{\max _{t}\left|x_{1,2}(t)\right|} \times 100 \%, \frac{e_{3}}{\max _{t}\left|x_{2,1}(t)\right|} \times 100 \%, \frac{e_{4}}{\max _{t}\left|x_{2,2}(t)\right|}\right.$

$\times 100 \%)$. Besides, we can observe that $e_{i}=0$ is not an equilibrium of the synchronization error dynamics when the perturbation is present. The results above may not be very surprising. For small perturbations to the nominal adjacency matrix corresponding to the coupling, one would intuitively expect the solutions remain almost synchronized within the clusters. However, this intuition may also fail, for instance, if the system is close to a bifurcation point. Therefore, it is important to develop tools that guarantee the required robustness of the synchronization with respect to the perturbation. In Sec. IV, this situation with perturbation on coupling impacting the partial synchronization of delay-coupled systems is generalized and forms the main problem addressed in this paper.

\section{PROBLEM FORMULATION}

In this section, we introduce the concept of practical partial synchronization, addressing the case where there are perturbations on the coupling. In addition, the synchronization error dynamics are also derived, which lay the basis for the stability analysis in Sec. IV A.

\section{A. Practical partial synchronization}

Suppose that there exists a viable partition $\mathscr{P}$ for the network of systems represented by the graph $\mathscr{G}=\left(\mathscr{V}, \mathscr{E}, A_{n}\right)$ and assume that the adjacency matrix of the network is perturbed to $A=A_{n}+\Delta A$, with perturbation bound

$$
\|\Delta A\|_{\infty} \leq \delta
$$



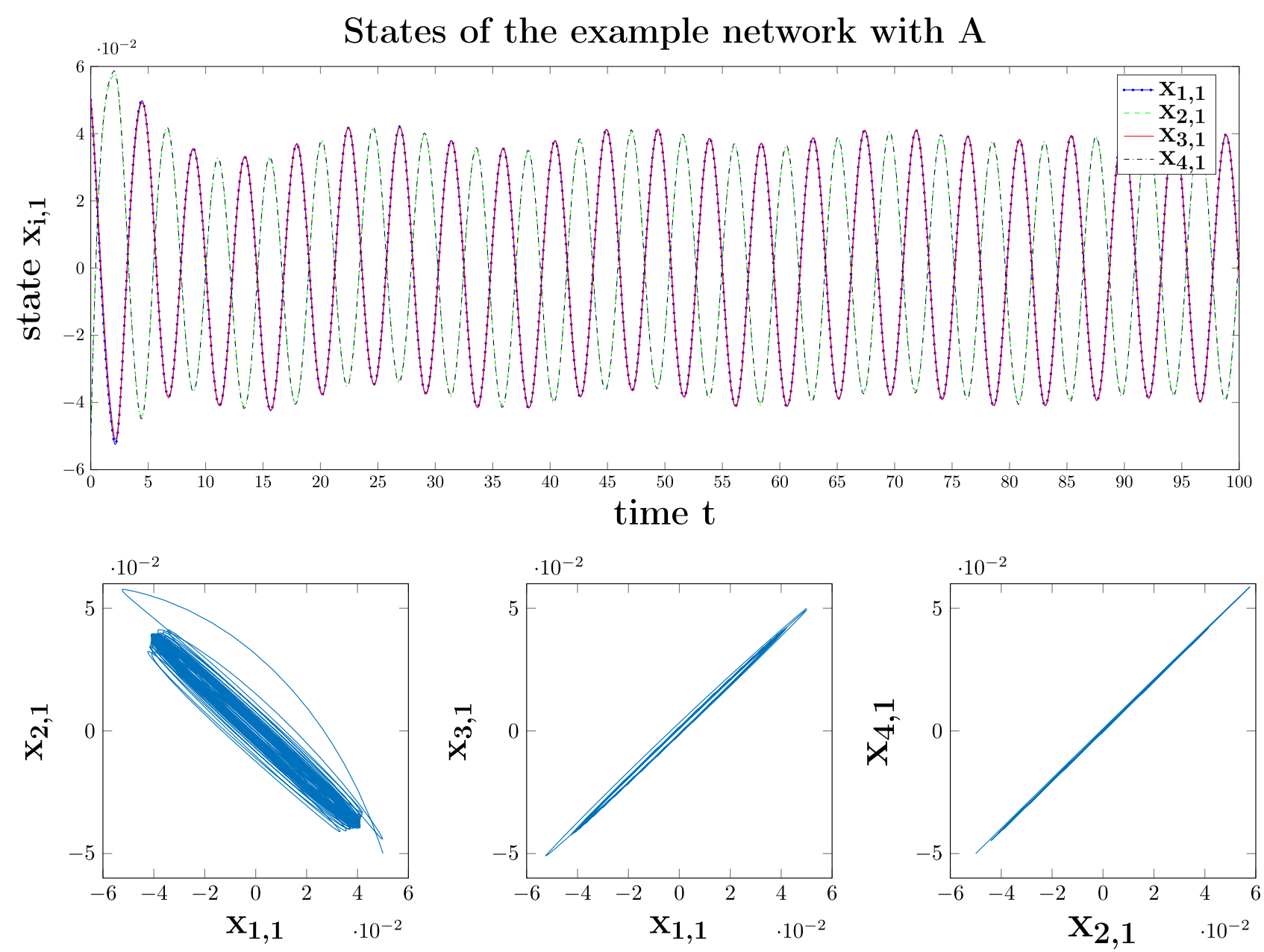

FIG. 4. Simulation of the network corresponding to Example 1, with adjacency matrix $A$.

where $\|\cdot\|_{\infty}$ denotes the $\infty$ induced matrix norm [the $\infty$ induced norm of matrix $A=\left(a_{i j}\right) \in \mathbb{R}^{N \times N}$ is defined as $\|A\|_{\infty}=$ $\left.\max _{1 \leq i \leq N} \sum_{j=1}^{N}\left|a_{i j}\right|\right]$. Note that the perturbed adjacency matrix $A$ should preserve the basic properties of the adjacency matrix: nonnegative entries and zero diagonal elements. That is, $\Delta A$ is constrained such that $a_{i j}^{\Delta}=0$ if $i=j$ and $a_{i j}^{\Delta} \geq-a_{i j}^{n}$ if $i \neq j$ with $a_{i j}^{\Delta}$ being the entries of $\triangle A$ and $a_{i j}^{n}$ the entries of $A_{n}$.

In this case, the row-sum conditions in Theorem 1 may be violated even for arbitrarily small perturbations, and exact partial synchronization may become impossible. However, using the following relaxation of the set $\mathscr{M}(\Pi)$ for $\mu \geq 0$ :

$$
\begin{aligned}
\mathscr{M}_{\mu}(\Pi):= & \left\{\phi \in \mathscr{C}\left([-\tau, 0], \mathbb{R}^{N n}\right) \mid \phi(\theta)=\operatorname{col}\left(\phi_{1}(\theta),\right.\right. \\
& \left.\ldots, \phi_{N}(\theta)\right), \phi_{i}(\theta) \in \mathbb{R}^{n}, i=1, \ldots, N, \| \phi(\theta) \\
& \left.-\left(\Pi \otimes I_{n}\right) \phi(\theta) \| \leq \mu, \forall \theta \in[-\tau, 0]\right\},
\end{aligned}
$$

we can analyze an approximate form of partial synchronization, formally defined as follows:

Definition 3. For a network of systems (1) and (2) represented by the graph $\mathscr{G}=\left(\mathscr{V}, \mathscr{E}, A_{n}\right)$, given a partition $\mathscr{P}$, permutation matrix $\Pi$, and perturbation bound $\delta$ in (13), the network is practically partially synchronized with respect to the clustering represented by $\mathscr{P}$ if there exist a class $\mathscr{K}$ [a continuous function $\beta:[0, a) \rightarrow \mathbb{R}_{\geq 0}$ is of class $\mathscr{K}$ if it is strictly increasing and $\beta(0)=0$; Khalil, 2001] function $\beta(\delta)$ and a function $T=T(\phi, \delta)$ such that whenever the adjacency matrix is perturbed to $A_{n}+\Delta A$ with $\|\Delta A\|_{\infty} \leq \delta$, the solutions satisfy

$$
x_{t}(\phi) \in \mathscr{M}_{\mu}(\Pi), \quad \forall \phi \in \mathscr{C}\left([-\tau, 0], \mathbb{R}^{N n}\right), \forall t>T,
$$

where $\mu=\beta(\delta)$.

Note that since $\beta \in \mathscr{K}, \mathscr{M}_{\mu=0}(\Pi)=\mathscr{M}(\Pi)$; hence, practical partial synchronization implies partial synchronization of the nominal network. 


\section{Synchronization errors of the example network}
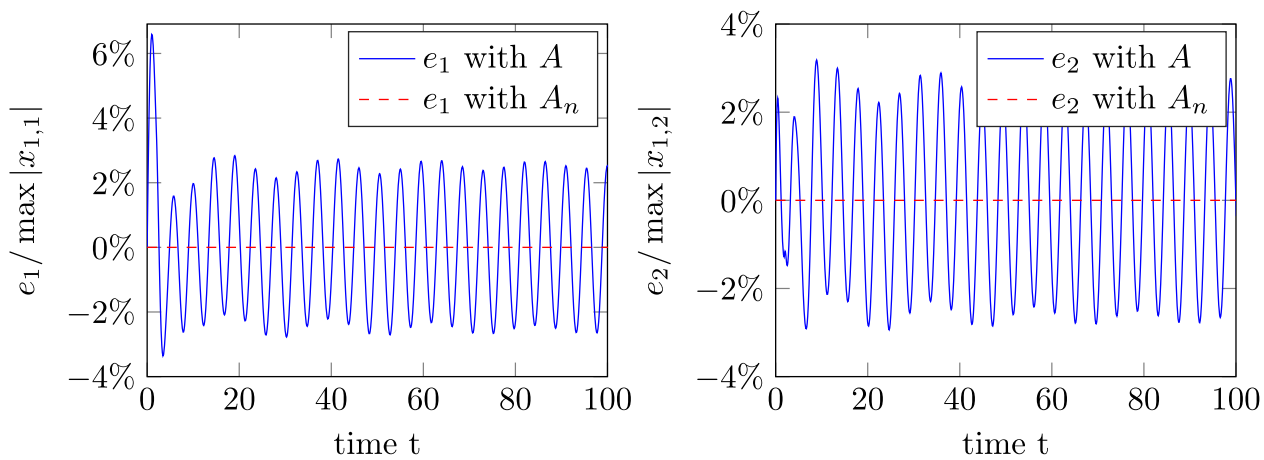

FIG. 5. Synchronization errors of the network corresponding to Example 1, with $A$ and $A_{n}$.

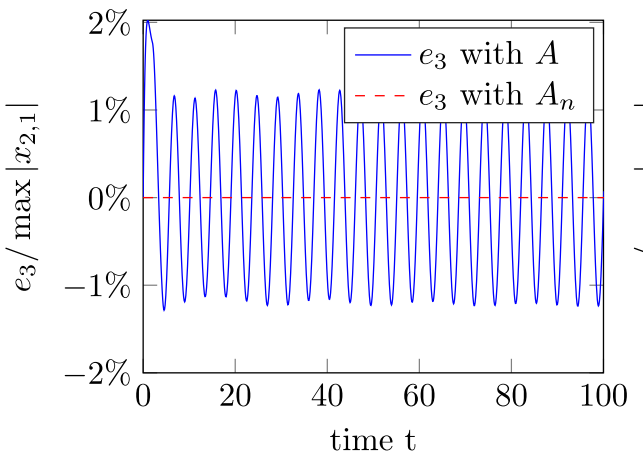

\section{B. Synchronization error dynamics}

Here, the synchronization error dynamics are derived for both nominal and perturbed networks. By comparing the synchronization error dynamics of these two cases, the impacts of the perturbations are shown, which explain why exact partial synchronization becomes impossible in the presence of perturbations.

\section{Nominal network}

Let us start with the nominal network using the procedure in Su et al. (2018). Suppose that there exists a viable partition $\mathscr{P}$ for the networked systems with the graph $\mathscr{G}=\left(\mathscr{V}, \mathscr{E}, A_{n}\right)$. Without loss of generality, we assume that the systems have been preordered into clusters according to the partition $\mathscr{P}$ associated with $\Pi$ as follows:

$$
\begin{array}{cc}
x_{1,1}, x_{1,2}, \ldots, x_{1, \kappa_{1}} & \text { cluster } 1, \\
x_{2,1}, x_{2,2}, \ldots, x_{2, \kappa_{2}} & \text { cluster } 2, \\
\vdots & \vdots \\
x_{\kappa, 1}, x_{\kappa, 2}, \ldots, x_{\kappa, \kappa_{\kappa}} & \text { cluster } \kappa .
\end{array}
$$

If we give the systems associated with $x_{1,1}, \ldots, x_{\kappa, 1}$, the role of reference systems of their clusters, we can define the synchronization errors by

$$
E_{i}=\left[\begin{array}{c}
e_{i, 2} \\
\vdots \\
e_{i, \kappa_{i}}
\end{array}\right]=\left[\begin{array}{c}
x_{i, 2}-x_{i, 1} \\
\vdots \\
x_{i, \kappa_{i}}-x_{i, 1}
\end{array}\right], \quad i=1, \ldots, \kappa .
$$

We denote $R_{i, j}$ as the row-sum of the first row of $i j$ th block of the adjacency matrix for $i, j \in\{1, \ldots, \kappa\}$. Note that these blocks have constant row-sums for the viable partition $\mathscr{P}$. Besides, the nodes in each cluster host the same dynamical system. We denote the dynamics of the nodes in cluster $i$ by $\tilde{f}_{i}, \tilde{B}_{i}, \tilde{C}_{i}, i=1,2, \ldots, \kappa$, where $\tilde{f}_{1}=f_{1}=f_{2}=\cdots=f_{\kappa_{1}}, \tilde{B}_{1}=B_{1}=B_{2}=\cdots=B_{\kappa_{1}}, \tilde{C}_{1}=C_{1}$ $=C_{2}=\cdots=C_{\kappa_{1}}, \quad \tilde{f}_{2}=f_{\kappa_{1}+1}=f_{\kappa_{1}+2}=\cdots=f_{\kappa_{1}+\kappa_{2}}, \quad \tilde{B}_{2}=B_{\kappa_{1}+1}$ $=B_{\kappa_{1}+2}=\cdots=B_{\kappa_{1}+\kappa_{2}}, \tilde{C}_{2}=C_{\kappa_{1}+1}=C_{\kappa_{1}+2}=\cdots=C_{\kappa_{1}+\kappa_{2}}$, and so on.

Letting

$$
R_{i}=\sum_{j=1}^{\kappa} R_{i, j}, \quad i=1, \ldots, \kappa,
$$

$\mathscr{X}(t)=\left[\begin{array}{c}E_{1}(t) \\ \vdots \\ E_{\kappa}(t)\end{array}\right] \in \mathbb{R}^{N n-\kappa n}, \quad \mathscr{X}_{r}(t)=\left[\begin{array}{c}\mathbf{1}_{\kappa_{1}-1} \otimes x_{1,1}(t) \\ \vdots \\ \mathbf{1}_{\kappa_{\kappa}-1} \otimes x_{\kappa, 1}(t)\end{array}\right] \in \mathbb{R}^{N n-\kappa n}$,

the error dynamics of the nominal network can be expressed as

$$
\dot{X}(t)=F\left(\mathscr{X}(t), \mathscr{X}_{r}(t)\right)-k A_{0} \mathscr{X}(t)+k A_{1} \mathscr{X}(t-\tau),
$$

where $F, A_{0}$, and $A_{1}$ are specified in Appendix A. Roughly speaking, $F$ is related to the differences of the nonlinear terms $f_{i}$ between the systems in each cluster, $A_{0}$ is related to the dynamics of the reference systems, and $A_{1}$ is related to the network structure. Note that $F\left(0, \mathscr{X}_{r}(t)\right)=0$. 
As a consequence of the viability of the partition, $\mathscr{X}(t)=0$ is an equilibrium point of the synchronization error dynamics (15). More specifically, because of the row-sum conditions and condition (9) in Lemma 1 , the other two terms containing $\mathscr{X}_{r}(t)$ and $\mathscr{X}_{r}(t-\tau)$, respectively, are canceled during the derivation of (15), which are not zero when $\mathscr{X}(t)=0$. The stability of partial synchronization manifolds can be inferred from the stability of this equilibrium, which is necessary for networks to exhibit exact partial synchronization in practice.

\section{Perturbed network}

Let us now look into the effect of the perturbation in (13) on the synchronization error dynamics (15). In the presence of the perturbation, the row-sums of the blocks in (8) are not anymore constant. Let us keep associating $x_{1,1}, \ldots, x_{\kappa, 1}$ with the role of reference systems. Hence, we continue to denote $R_{i, j}$ as the row-sum of the first row of $i j$ th block of the adjacency matrix for $i, j \in\{1, \ldots, \kappa\}$, while the sums of the other rows becomes $R_{i j}+\Delta_{i j, l}$ with $l=2, \ldots, \kappa_{i}$. That is, for the block $A_{i j}$ in (8), its row-sums are denoted by

$$
\left[\begin{array}{c}
R_{i j} \\
R_{i j}+\Delta_{i j, 2} \\
\vdots \\
R_{i j}+\Delta_{i j, \kappa_{i}}
\end{array}\right],
$$

where by the assumption that $\|\Delta A\|_{\infty} \leq \delta, \quad\left|\Delta_{i j, l}\right| \leq \delta$ for $i$, $j=1, \ldots, \kappa, l=1, \ldots, \kappa_{i}$. The collection of $\Delta_{i j, l}$ is denoted by $\Delta \in$ $\mathbb{R}^{(N-\kappa) \times \kappa}$ as below

$$
\Delta=\left[\begin{array}{cccc}
\Delta_{11,2} & \Delta_{12,2} & \cdots & \Delta_{1 \kappa, 2} \\
\vdots & \vdots & \vdots & \vdots \\
\Delta_{11, \kappa_{1}} & \Delta_{12, \kappa_{1}} & \cdots & \Delta_{1 \kappa, \kappa_{1}} \\
\vdots & \ddots & \ddots & \vdots \\
\Delta_{\kappa 1,2} & \Delta_{\kappa 2,2} & \cdots & \Delta_{\kappa \kappa, 2} \\
\vdots & \vdots & \vdots & \vdots \\
\Delta_{\kappa 1, \kappa_{1}} & \Delta_{\kappa 2, \kappa_{1}} & \cdots & \Delta_{\kappa \kappa, \kappa_{1}}
\end{array}\right] .
$$

Then, the synchronization error dynamics can be expressed as

$$
\begin{aligned}
\dot{X}(t)= & F\left(\mathscr{X}(t), \mathscr{X}_{r}(t)\right)-k A_{0} \mathscr{X}(t)+k A_{1} \mathscr{X}(t-\tau) \\
& +n\left(\mathscr{X}_{r}(t), \mathscr{X}_{r}(t-\tau), \Delta\right),
\end{aligned}
$$

where the additional term compared to (15) is given by

$$
n\left(\mathscr{X}_{r}(t), \mathscr{X}_{r}(t-\tau), \Delta\right)=A_{2}(\Delta) \mathscr{X}_{r}(t)+A_{3}(\Delta) \mathscr{X}_{r}(t-\tau),
$$

with $A_{2}(\Delta)$ and $A_{3}(\Delta)$ specified in Appendix A, which satisfies the property

$$
n\left(\mathscr{X}_{r}(t), \mathscr{X}_{r}(t-\tau), 0\right)=0 .
$$

As a consequence of Assumption 1, all solutions of systems (1), coupled via (2), converge to a positively invariant set $\Omega$. Hence, without loosing generality, we can assume that the initial conditions satisfy $x_{0} \in \Omega$, implying $x_{t} \in \Omega$ for all $t \geq 0$. Meanwhile, $\left\|A_{2}\right\|$ and $\left\|A_{3}\right\|$ depend on $\Delta$, in such a way that they can be uniformly bounded for all $x_{t} \in \Omega$. Therefore, we can assume that the perturbation term is also bounded,

$$
\left\|n\left(\mathscr{X}_{r}(t), \mathscr{X}_{r}(t-\tau), \Delta\right)\right\| \leq \gamma,
$$

where $\gamma=\eta(\delta)$ and function $\eta$ belongs to class $\mathscr{K}$. Furthermore, there exists a bounded set $\Omega_{r}$, independent of $\delta$ such that $x_{t} \in \Omega$ implies $\mathscr{X}_{r} \in \Omega_{r}$.

In summary, the perturbation on the coupling introduces an additional bounded item in the equation for the synchronization error dynamics. The item becomes zero, in general, if and only if the perturbation becomes zero. Moreover, due to this term, the zero point is generally not an equilibrium of the synchronization error dynamics. All the above allow us to connect the problem of practical partial synchronization with practical stability of the error system, in the sense of Definition 1 in Villafuerte et al. (2011).

Definition 4. Given a partition $\mathscr{P}$, permutation matrix $\Pi$, and perturbation bound $\gamma$, the network is practically partially synchronized with the clustering represented by $\mathscr{P}$ if system (18) with $\mathscr{X}_{r} \in \Omega_{r}$ and the initial data $\mathscr{X}_{0}=\Psi$ is globally practically stable, i.e., there exist functions $\beta(\gamma) \in \mathscr{K}$ and $T=T(\gamma, \Psi) \geq 0$ such that, whenever $\|n(t)\| \leq \gamma$ and $\mathscr{X}_{r}(t) \in \Omega_{r}$ for all $t \geq 0$, we have

$\|\mathscr{X}(\Psi)(t)\| \leq \beta(\gamma), \quad \forall \Psi \in \mathscr{C}\left([-\tau, 0], \mathbb{R}^{(N-\kappa) n}\right), \quad \forall t \geq T(\gamma, \Psi)$.

In Sec. $V$, we derive tractable sufficient conditions for practical stability of (18), accompanied by the explicit construction of function $\beta$. It is important to note that the "additive perturbation" $n$ in (18) perturbs the zero equilibrium point. As a distinctive feature of this work, this in turn implies that in the stability analysis, a linearization around the equilibrium, which is a cornerstone in most works on exact (partial) synchronization, is no longer possible.

\section{MAIN RESULTS}

In this section, sufficient conditions for practical partial synchronization are derived through the stability analysis of the synchronization error dynamics. A Lyapunov(-Krasovskii) theorem is used for the stability analysis and the obtained conditions are expressed in form of LMIs. Whether or not the LMIs are satisfied can be efficiently determined with numerical tools.

As mentioned above, the solutions of coupled systems (1) and (2) are bounded, that is, $x \in \Omega$ with $\Omega$ being a bounded set. Hence, for every cluster, we have $x_{i, j} \in \Omega_{x}, i=1, \ldots, \kappa, j=1, \ldots, \kappa_{i}$ with $\Omega_{x}$ being a bounded set induced by $\Omega$. Additionally, the functions $f_{i}$ are sufficiently smooth. Therefore, a Lipschitz condition [A function $f: \Omega \rightarrow \mathbb{R}^{n}$ satisfies a Lipschitz condition if there exists a $L \in \mathbb{R}_{+}$ such that $\|f(x)-f(y)\| \leq L\|x-y\|, \forall x, y \in \Omega$. A Lipschitz condition is satisfied for any smooth function $f(x), x \in \Omega$ with $\Omega$ being a bounded set.] is satisfied for each component of $F\left(\mathscr{X}(t), \mathscr{X}_{r}(t)\right)$,

$$
\left\|\tilde{f}_{i}\left(x_{i, j}+e_{i, j}\right)-\tilde{f}_{i}\left(x_{i, j}\right)\right\| \leq L_{i, j}\left\|e_{i, j}\right\|, \quad L_{i, j} \in \mathbb{R}_{+}, \forall x_{i, j}+e_{i, j}, x_{i, j} \in \Omega_{x} .
$$

Combining (23) with the structure of (A1), we can also conclude that there exists an $L \in \mathbb{R}_{+}$such that for all possible $\mathscr{X}_{r}(t)$ and $\mathscr{X}(t)$,

$$
\left\|F\left(\mathscr{X}, \mathscr{X}_{r}\right)\right\| \leq L\|\mathscr{X}\| \text {. }
$$


Using the above Lipschitz condition, we extend the method in Villafuerte et al. (2011) to analyze the practical stability of the time delay system (18), which includes a nonlinear component $F\left(\mathscr{X}(t), \mathscr{X}_{r}(t)\right)$. In Villafuerte et al. (2011), only linear time-delay systems are considered. Through this stability analysis, the conditions for practical partial synchronization are derived, which also yield an explicit expression of $\mu$ as a $\mathscr{K}$ function of $\gamma$, as stated in the theorem below.

Theorem 1. Consider a network of systems (1) and (2) represented by the graph $\mathscr{G}=(\mathscr{V}, \mathscr{E}, A)$. For a partition $\mathscr{P}$, if there exist positive definite matrices $P, Q \in \mathbb{R}^{(N-\kappa) n \times(N-\kappa) n}$ and a positive constant $\sigma$ such that the inequalities (for a symmetric matrix $M$, the inequalities $M<0$ and $M \leq 0$ denote that $M$ is negative definite, respectively, negative semidefinite)

$$
\begin{gathered}
\mathscr{M}(P, Q)+2 \sigma \mathscr{N}(P)<0, \\
L<\frac{\lambda_{\min }(P)}{\lambda_{\max }(P)} \sigma,
\end{gathered}
$$

hold, where

$$
\mathscr{M}(P, Q)=\left[\begin{array}{cc}
-k P A_{0}-k A_{0}^{\top} P+Q & k P A_{1} \\
k A_{1}^{\top} P & -e^{-2 \sigma \tau} Q
\end{array}\right], \quad \mathscr{N}(P)=\left[\begin{array}{cc}
P & 0 \\
0 & 0
\end{array}\right]
$$

and $L$ is the Lipschitz gain defined in (24), then error system (18), corresponding to this partition, is globally practically stable, i.e., the network is practically partially synchronized with respect to $\mathscr{P}$.

Furthermore, functions $\beta$ and $T$ in (22) can be constructed from the solutions of (25)-(27) (see Appendix B).

The proof of Theorem 1 is given in Appendix B. In the proof, the Lyapunov-Krasovskii method is used, which involves a "energy" functional called Lyapunov-Kraosvskii functional $v\left(\mathscr{X}_{t}\right)$. As its naming indicates, $v\left(\mathscr{X}_{t}\right)$ is a function of the functional state $\mathscr{X}_{t}$. Similar to a Lyapunov function for a delay-free system, the LyapunovKrasovskii functional should be positive definite and its derivative with respect to time along the trajectories of the system should be negative definite. A typical Lyapunov-Kraosvskii functional includes a quadratic term and some integral terms. Depending the choice of the integral terms, delay-independent or delay-dependent stability conditions can be derived. To prove Theorem 1, the following Lyapunov-Krasovskii functional is chosen, which provides delaydependent conditions:

$$
v\left(\mathscr{X}_{t}\right)=\mathscr{X}^{\top}(t) P \mathscr{X}(t)+\int_{-\tau}^{0} \mathscr{X}^{\top}(t+\theta) e^{2 \sigma \theta} \mathrm{Q} \mathscr{X}(t+\theta) \mathrm{d} \theta .
$$

We refer to Appendix B for the details.

Although the LMIs are not very transparent, in the sense that they do not explicitly reveal a relation between practical partial synchronization and the network parameters ( $k$ and $\tau$ ), some conclusions can still be drawn by looking into the structure of the LMIs. For exact partial synchronization, it has been shown in Steur et al. (2012) that there exist positive constants $\bar{k}$ and $\bar{\rho}$ such that if $k>\bar{k}$ and $k \tau<$ $\bar{\rho}$, the exact partial synchronization manifold is stable, as illustrated in Fig. 6. Similar conclusions are also expected for practical partial synchronization. First, $k$ should be large enough to achieve practical partial synchronization. From the item $-k P A_{0}-k A_{0}^{\top} P+Q$ at the

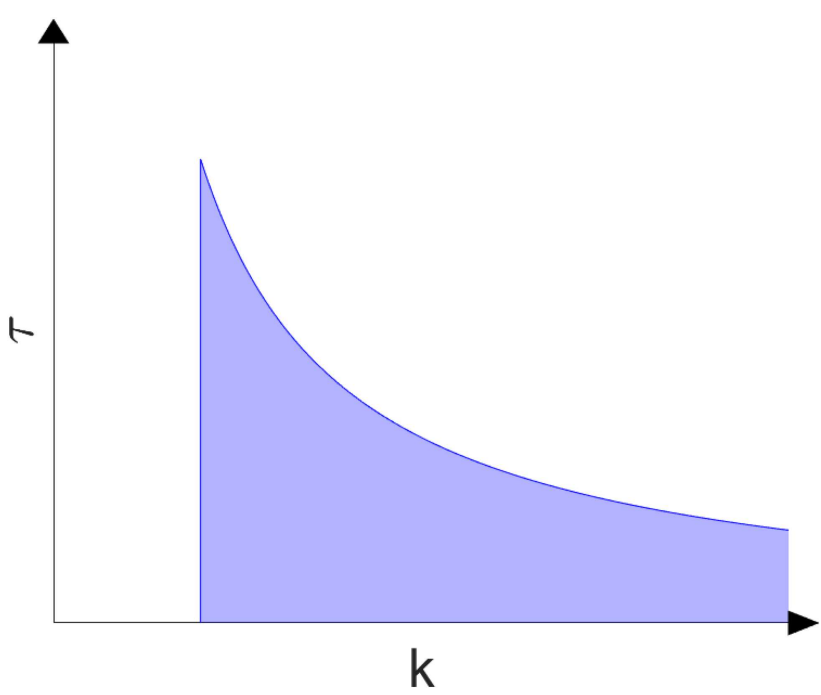

FIG. 6. Exact partial synchronization region (blue-colored area) in $(k, \tau)$ space (Steur et al., 2012).

upper-left corner of $\mathscr{M}(P, Q)$, we can see that a larger $k$ can contribute to the negative definiteness of $\mathscr{M}(P, Q)$. Second, $k$ should also be limited. Let us focus on $\mathscr{M}(P, Q)$. For $\mathscr{M}(P, Q)$ to be negative definite, the Schur complement of $-e^{-2 \sigma \tau} Q$ should also be negative definite, i.e.,

$$
-k P A_{0}-k A_{0}^{\top} P+Q+k^{2} P A_{1} e^{2 \sigma \tau} Q^{-1} A_{1}^{\top} P<0 .
$$

Note that the last term contains $k^{2}$. Therefore, when $k$ is too large, the last item becomes dominant, thus making the above inequality difficult to fulfill. Third, $\tau$ can be small. A smaller $\tau$ helps the term $-e^{-2 \sigma \tau} Q$ in $\mathscr{M}(P, Q)$ to remain negative definite. Finally, large $\tau$ is not desired. Clearly, for large $\tau,-e^{-2 \sigma \tau} Q$ goes to zero, thus making LMIs (25) difficult to be satisfied.

In the derivation of the analysis conditions in Theorem 1, we have included all information about the nonlinearity of functions $f_{i}$ into a single Lipschitz gain which needs to be compensated by the coupling. This approach facilitates the formulation of analysis conditions; however, it also leads to the additional restriction on the upper bound of $L$ in (26), which indicates that Theorem 1 is applicable for systems with mild nonlinearity. To reduce the restriction, we propose another theorem where more information on (the boundedness of) the nonlinearity is exploited. To this end, we introduce the bounds of the nonlinearity componentwise such that the boundedness of the nonlinearity is captured by a series of bounds instead of a single constant $L$.

Let us reformulate $\mathscr{X}(t), \mathscr{X}_{r}(t)$ and $F\left(\mathscr{X}(t), \mathscr{X}_{r}(t)\right)$ as follows:

$$
\mathscr{X}(t)=\left[\begin{array}{c}
\mathscr{X}_{1}(t) \\
\vdots \\
\mathscr{X}_{N n-\kappa n}(t)
\end{array}\right], \quad \mathscr{X}_{r}(t)=\left[\begin{array}{c}
\mathscr{X}_{r, 1}(t) \\
\vdots \\
\mathscr{X}_{r, N n-\kappa n}(t)
\end{array}\right],
$$




$$
F\left(\mathscr{X}(t), \mathscr{X}_{r}(t)\right)=\left[\begin{array}{c}
\psi_{1}\left(\mathscr{X}_{1}(t), \mathscr{X}_{r}(t)\right) \\
\vdots \\
\psi_{N n-\kappa n}\left(\mathscr{X}_{N n-\kappa n}(t), \mathscr{X}_{r, N n-\kappa n}(t)\right)
\end{array}\right],
$$

where $\mathscr{X}_{\ell}(t), \mathscr{X}_{r, \ell}(t) \in \mathbb{R} \quad$ and $\quad \psi_{\ell}: \mathbb{R} \times \mathbb{R} \rightarrow \mathbb{R}, \quad \ell=1, \ldots$, $\mathrm{N} n-\kappa n$.

Since functions $f_{i}$ are sufficiently smooth and the solutions $x_{i}(t)$ are bounded, there exist $F_{\ell}^{-}$and $F_{\ell}^{+}$such that

$$
F_{\ell}^{-} \leq \frac{\psi_{\ell}\left(\mathscr{X}_{\ell}(t), \mathscr{X}_{r, \ell}(t)\right)}{\mathscr{X}_{\ell}(t)} \leq F_{\ell}^{+}, \quad \text { for } \ell=1, \ldots, N n-\kappa n,
$$

or equivalently,

$$
\left(\psi_{\ell}\left(\mathscr{X}_{\ell}(t), \mathscr{X}_{r, \ell}(t)\right)-F_{\ell}^{-} \mathscr{X}_{\ell}(t)\right)\left(\psi_{\ell}\left(\mathscr{X}_{\ell}(t), \mathscr{X}_{r, \ell}(t)\right)-F_{\ell}^{+} \mathscr{X}_{\ell}(t)\right) \leq 0 \text {. }
$$

With $F_{\ell}^{+}$and $F_{\ell}^{-}$specified, another set of conditions for practical partial synchronization is provided in the theorem below.

Theorem 2. Consider a network of systems (1) and (2) represented by the graph $\mathscr{G}=(\mathscr{V}, \mathscr{E}, A)$. For a partition $\mathscr{P}$, if there exist symmetric positive definite matrices $P, Q, Z, W \in \mathbb{R}^{(N-\kappa) n \times(N-\kappa) n}, a$ positive definite diagonal matrix $V \in \mathbb{R}^{(N-\kappa) n \times(N-\kappa) n}$, and a positive constant $\sigma$ such that the inequality

$$
\Theta+H_{1} P H_{2}+H_{2}^{\top} P H_{1}^{\top}+\tau^{2} H_{2}^{\top} Z H_{2}<0
$$

holds, where

$$
\Theta=\left[\begin{array}{cccc}
2 \sigma P+Q-e^{-2 \sigma \tau} Z-F_{1} V & e^{-2 \sigma \tau} Z & F_{2} V & 0 \\
* & -e^{-2 \sigma \tau} Q-e^{-2 \sigma \tau} Z & 0 & 0 \\
* & * & -V & 0 \\
* & * & * & -W
\end{array}\right],
$$

$H_{1}=\left[\begin{array}{llll}I & 0 & 0 & 0\end{array}\right]^{\top}$,

$H_{2}=\left[\begin{array}{llll}-k A_{0} & k A_{1} & I & I\end{array}\right]$,

$F_{1}=\operatorname{diag}\left\{F_{1}^{-} F_{1}^{+}, F_{2}^{-} F_{2}^{+}, \ldots, F_{N n-\kappa n}^{-} F_{N n-\kappa n}^{+}\right\}$,

$F_{2}=\operatorname{diag}\left\{\frac{F_{1}^{-}+F_{1}^{+}}{2}, \frac{F_{2}^{-}+F_{2}^{+}}{2}, \ldots, \frac{F_{N n-\kappa n}^{-}+F_{N n-\kappa n}^{+}}{2}\right\}$,

then the corresponding system (18) is globally practically stable. That is, the network is practically partially synchronized with respect to $\mathscr{P}$. Furthermore, functions $\beta$ and $T$ in (22) can be chosen as for (1) if $\|\Psi\|_{s} \leq \frac{\gamma}{\sqrt{2 \sigma \alpha_{2} \lambda_{\min }(W)}}$, then

$$
\mu=\beta(\gamma)=\frac{\gamma}{\sqrt{2 \sigma \alpha_{1} \lambda_{\min }(W)}}, \quad T=0 ;
$$

for (2) if $\|\Psi\|_{s}>\frac{\gamma}{\sqrt{2 \sigma \alpha_{2} \lambda_{\min }(W)}}$, then

$$
\begin{aligned}
& \mu=\beta(\gamma)=\frac{k_{\mu} \gamma}{\sqrt{2 \sigma \alpha_{1} \lambda_{\min }(W)}}, \\
& T=\frac{1}{2 \sigma} \ln \left(\frac{2 \sigma \alpha_{2}\|\Psi\|_{s}^{2}-\gamma^{2} \lambda_{\max }(W)}{2 \sigma \alpha_{1} \mu^{2}-\gamma^{2} \lambda_{\max }(W)}\right),
\end{aligned}
$$

with $1<k_{\mu}<\frac{1}{\gamma} \sqrt{\frac{2 \sigma \alpha_{2}}{\lambda_{\max }(W)}}\|\Psi\|_{s}$.

Here, $\|\Psi\|_{s}$ is the supremum norm of the initial data $\|\Psi(\theta)\|_{s}:=$ $\sup _{\theta \in[0-\tau]}\|\Psi(\theta)\| \quad \alpha_{1}=\lambda_{\text {min }}(P), \quad \alpha_{2}=\lambda_{\max }(P)+\tau \lambda_{\max }(Q)+$ $\tau \lambda_{\max }(Z)$, and $*$ denotes the symmetric terms in symmetric matrices.
The proof of Theorem 2 is given in Appendix C. As with the proof of Theorem 1, the Lyapunov-Krasovskii method is used. A Lyapunov Krassovskii (energy) functional of the following form is used:

$$
\begin{aligned}
v\left(\mathscr{X}_{t}\right)= & \mathscr{X}^{\top}(t) P \mathscr{X}(t)+\int_{t-\tau}^{t} \mathscr{X}^{\top}(\theta) e^{2 \sigma(\theta-t)} Q \mathscr{X}(\theta) \mathrm{d} \theta \\
& +\tau \int_{-\tau}^{0} \int_{t+s}^{s} \dot{\mathscr{X}}^{\top}(\theta) e^{2 \sigma(\theta-t)} Z \dot{\mathscr{X}}(\theta) \mathrm{d} \theta \mathrm{d} s .
\end{aligned}
$$

We refer to Appendix $\mathrm{C}$ for the details.

As with Theorem 1, similar conclusions on the parametric dependence of the practical partial synchronization can also be drawn from Theorem 2. Let us focus the block at the upper-left corner of the matrix $\Theta+H_{1} P H_{2}+H_{2}^{\top} P H_{1}^{\top}+\tau^{2} H_{2}^{\top} Z_{2}$, which is given by

$$
-k P A_{0}-k A_{0}^{\top} P+\tau^{2} k^{2} A_{0}^{T} Z A_{0}+2 \sigma P+Q-e^{-2 \sigma \tau} Z-F_{1} V .
$$

For the LMIs to be solvable, it is desired for this matrix to be negative definite. Apparently, we can see that a sufficiently large $k$ helps the matrix (37) to be negative definite due to the part $-k P A_{0}-k A_{0}^{\top} P$. However, due to the part $\tau^{2} k^{2} A_{0}^{T} Z A_{0}, k \tau$ should be limited such that (37) can be negative definite.

Remark 2: Similarly as in Villafuerte et al. (2011), an "exponential estimate" of the synchronization errors $\mathscr{X}$ is provided when deriving these two theorems, which indicates how fast solutions of the coupled systems converge to the set $\mathscr{M}_{\mu}$. $\sigma$ is related to the exponential decay rate estimate. A larger value of $\sigma$ indicates a faster decay of the synchronization errors. In addition, as can be seen from the expressions of $\beta(\gamma), \sigma$ is also inversely related to amplification of the perturbation. Thus, a larger value of $\sigma$ results in a smaller bound of the synchronization errors $\mu$. However, the LMIs (25) and (32) may become unsolvable (infeasible) for a large $\sigma$, due to the presence of such terms as $2 \sigma \mathscr{N}(P),-e^{-2 \sigma \tau} \mathrm{Q}$ in (25) and $2 \sigma P,-e^{-2 \sigma \tau} \mathrm{Z}$ in (32), respectively. Furthermore, because of the presence of the terms $-e^{-2 \sigma \tau} Q$ and $-e^{-2 \sigma \tau} Z$, a smaller value of time-delay $\tau$ is required for a larger value of $\sigma$. Therefore, there exists a trade-off when choosing the value of $\sigma$. A practical way to handle this trade-off, when using the theorems, is to start with a small $\sigma$ for the sake of the LMIs feasibility and then increase it incrementally to a desired value (e.g., for a small enough bound of the synchronization errors $\mu$ ) for which the LMIs are still feasible with an acceptable time-delay $\tau$.

\section{NUMERICAL EXAMPLE}

In this section, we revisit Example 1 presented in Sec. III with the focus on the perturbed network, which is analyzed by using the results from Sec. V.

The network is shown in Fig. 7. Recall that the adjacency matrix is perturbed with $\Delta A$ such that

$$
A=A_{n}+\Delta A=\left[\begin{array}{cccc}
0 & 2 & 0.1 & 1 \\
4 & 0 & 2 & 0.3 \\
0.1 & 1 & 0 & 2 \\
3 & 0.3 & 3 & 0
\end{array}\right]+\left[\begin{array}{cccc}
0 & 0.2 & 0 & 0 \\
0.2 & 0 & 0 & 0.05 \\
0 & 0 & 0 & 0 \\
0.1 & 0 & 0.2 & 0
\end{array}\right] .
$$

It can be proved that systems (11) of the network are semipassive for any real value of parameter $\epsilon$ (see Appendix D). In what follows, 


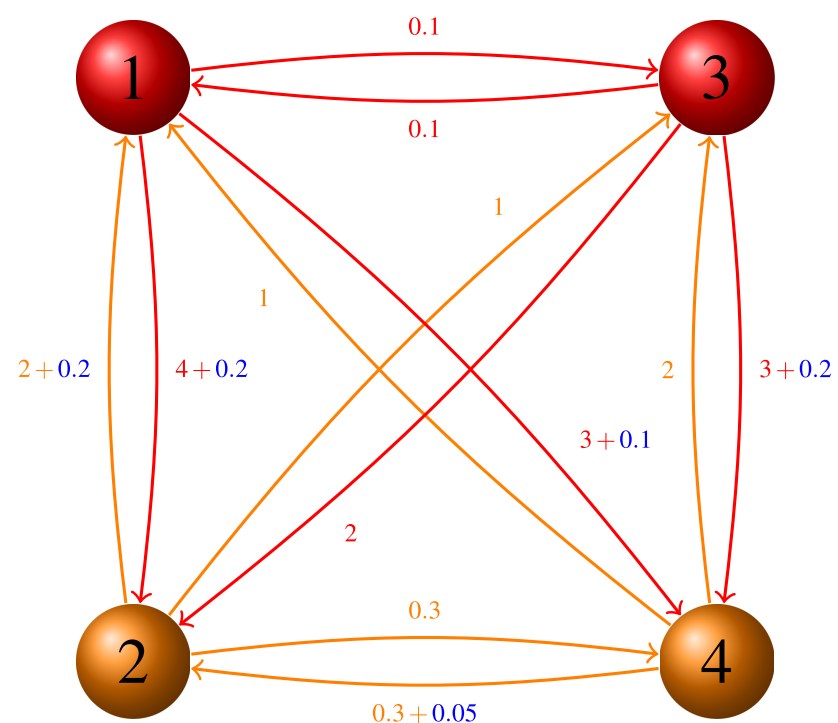

FIG. 7. The network of Example 1 with adjacency matrix $A$.

we take $\epsilon=-0.2$. Recall that for the nominal adjacency matrix $A_{n}$, partition $\mathscr{P}=\{\{1,3\},\{2,4\}\}$ is a viable partition. However, due to the perturbation $\Delta A$, no viable partition exists for this network with $A$. The corresponding synchronization error dynamics of form (18) are derived accordingly. The key matrices $A_{0}$ and $A_{1}$, which are required to construct the LMIs (32) in Theorem 2, are given by

$$
\begin{aligned}
& A_{0}:=\left[\begin{array}{cc}
R_{1} B C & 0 \\
0 & R_{2} B C
\end{array}\right]-\frac{1}{k}\left[\begin{array}{cc}
A_{s} & 0 \\
0 & A_{s}
\end{array}\right], \\
& A_{1}:=A_{\text {red }} \otimes B C,
\end{aligned}
$$

where

$$
\begin{aligned}
& R_{1}=3.3, \quad R_{2}=6.55 \\
& A_{s}=\left[\begin{array}{cc}
-\varepsilon & 1 \\
-1 & -\varepsilon
\end{array}\right]=\left[\begin{array}{cc}
0.2 & 1 \\
-1 & 0.2
\end{array}\right], \quad B=C^{\top}=\left[\begin{array}{ll}
0 & 1 \\
1 & 0
\end{array}\right], \\
& A_{\text {red }}=\left[\begin{array}{cc}
-0.1 & 1 \\
1.2 & -0.35
\end{array}\right] .
\end{aligned}
$$

Note that matrix $A_{s}$ represents the linear components of systems (11). Matrix $A_{0}$ is extended to include these linear components.

For a given pair $(k, \tau)$, we can use Theorem 2 to check if the network is practically partially synchronized in two steps: (1) estimate the values of $F_{\ell}^{+}$and $F_{\ell}^{-}$by using numerical simulations of the network and (2) check the feasibility of the LMIs in Theorem 2 for a given $\sigma$. As mentioned in Secs. II-VI, the states of the network dynamics converge to a positively invariant set in finite time. Therefore, in order to reduce conservatism, at the first step, only the data after some time are used for estimation. Here, we use the data after
600 time units for estimation. In particular, we find that the LMIs (32) are solvable for $k=0.3, \tau=2$ with $\sigma=0.22$ by using the MATLAB toolboxes of SDPT3 (Toh et al., 1999) and YALMIP (Lofberg, 2004). In detail, first, with this pair of $(k, \tau)$, we construct $F_{1}$ and $F_{2}$ using the estimated values of $F_{i}^{+}$and $F_{i}^{-}$given by

$$
\left[\begin{array}{l}
F_{1}^{+} \\
F_{2}^{+} \\
F_{3}^{+} \\
F_{4}^{+}
\end{array}\right]=\left[\begin{array}{c}
-0.0064 \\
0.0009 \\
1.4035 \\
0.0372
\end{array}\right], \quad\left[\begin{array}{l}
F_{1}^{-} \\
F_{2}^{-} \\
F_{3}^{-} \\
F_{4}^{-}
\end{array}\right]=\left[\begin{array}{l}
-0.0480 \\
-0.0255 \\
-0.0963 \\
-0.1279
\end{array}\right] .
$$

Now, the values of $H_{1}, H_{2}, F_{1}, F_{2}, \tau$, and $k$ are available for solving the LMIs. Second, with the help of the YALMIP and SDPT3 toolboxes, the LMIs in (32) are cast into an optimization problem with the matrices $P, Q, Z, W$, and $V$ as design variables, then MATLAB automatically construct the matrices if it finds such matrices exist for a given $\sigma$. To find the maximum possible value of $\sigma$, the second step is repeated for a series of values of $\sigma$ starting from 0.01 with an incremental step of 0.01 . It turns out that until $\sigma=0.22$, these matrices exist for $k=0.3, \tau=2$, and their values are presented in Appendix E. It is important to point out that for these values of $(k, \tau)$, the zero solution $\left(x_{i}=0\right)$ of the coupled network dynamics is (locally) exponentially unstable, which can be verified by checking the spectral abscissa (rightmost eigenvalues) of the linearized dynamics of the whole coupled network. Notice that there does not exist a partial synchronization manifold corresponding to full synchronization.

As a validation of the obtained results, we have done a simulation of the network with $k=0.3, \tau=2$, and the adjacency matrix $A$ defined in (38), using the dde23 solver from MATLAB with relative and absolute tolerance of $1 e^{-3}$ and $1 e^{-6}$, respectively. The simulation runs over 1000 time units. Unlike in Sec. III, initial data outside the partial synchronization manifold are used in this simulation such that we can also investigate the stability of partial synchronization. The values of the initial data are used are $x_{1}(\theta)=\left[\begin{array}{lll}0.0170 & 0.0365\end{array}\right]^{\top}, x_{2}(\theta)=\left[\begin{array}{lll}0.0035 & 0.0190\end{array}\right]^{\top}, x_{3}(\theta)=$ $\left[\begin{array}{lll}0.0208 & 0.0462\end{array}\right]^{\top}, x_{4}(\theta)=\left[\begin{array}{lll}0.0283 & 0.0378\end{array}\right]^{\top},-\tau \leq \theta \leq 0$, which are randomly chosen in the interval of $[0,0.05]$.

The evolution of the states $x_{i, 1}, i=1,2,3,4$ is depicted in Fig. 8 in the time interval $[0,100]$ (before partial synchronization is fully established) and Fig. 9 in the time interval $[990,1000]$ (after partial synchronization is fully established). As can be seen from those figures, $x_{1,1}$ and $x_{3,1}$, respectively, $x_{2,1}$ and $x_{4,1}$ converge to each other (up to a tolerance which is barely visible). In addition, Fig. 9 also shows the solutions converge to a limit cycle, which is induced by the nonlinear terms $-x_{i, 1}(t)\left(x_{i, 1}^{2}(t)+x_{i, 2}^{2}(t)\right)$ and $-x_{i, 2}(t)\left(x_{i, 1}^{2}(t)+\right.$ $\left.x_{i, 2}^{2}(t)\right)$ in (11). To compare exact synchronization with practical synchronization, we have also simulated the network with the same values of $(k, \tau)$ and the nominal adjacency matrix $A_{n}$. The synchronization errors $e_{1}=x_{3,1}-x_{1,1}, e_{2}=x_{3,2}-x_{1,2}, e_{3}=x_{4,1}-x_{2,1}$, and $e_{4}=x_{4,2}-x_{2,2}$ of the networks with $A$ and $A_{n}$ are shown in Figs. 10 and 11 . As in Fig. 5, the errors are scaled by the maximum magnitudes of the relevant variables in these figures. Clearly, the synchronization errors of the network with $A$ are larger than that of the network with $A_{n}$. Besides, the figures also indicate that the practically synchronized states only converge to each other up to some bound but cannot become exactly identical in the network with $A$. 


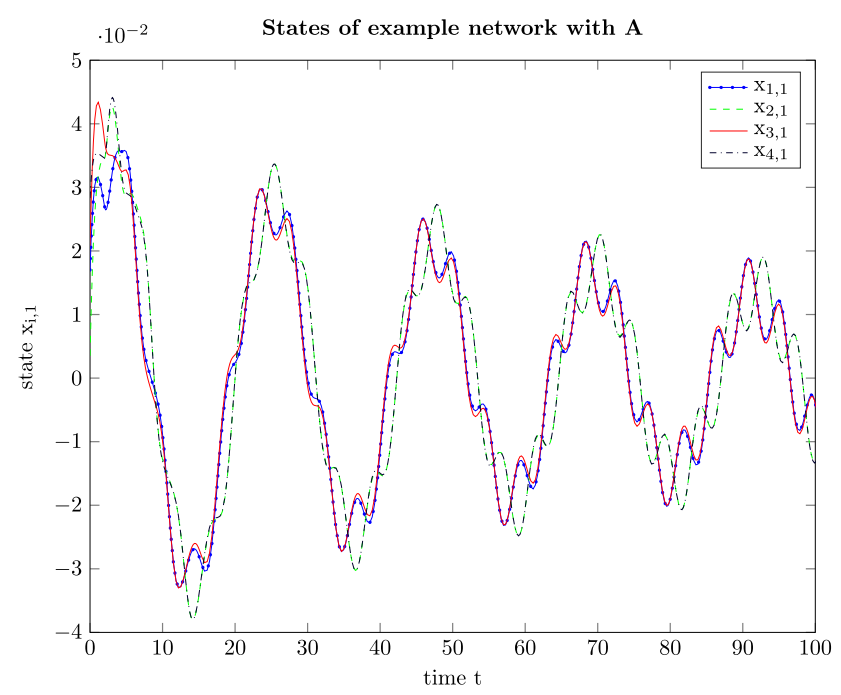

FIG. 8. The states $x_{i, 1}$ of the network with $k=0.3, \tau=2$ in the time interval $[0,100]$.

To demonstrate the relation between the size of the perturbation and the bound of the synchronization errors, we run a series of the simulations of this network with the same $k$ and $\tau$, but with a series of different adjacency matrices defined

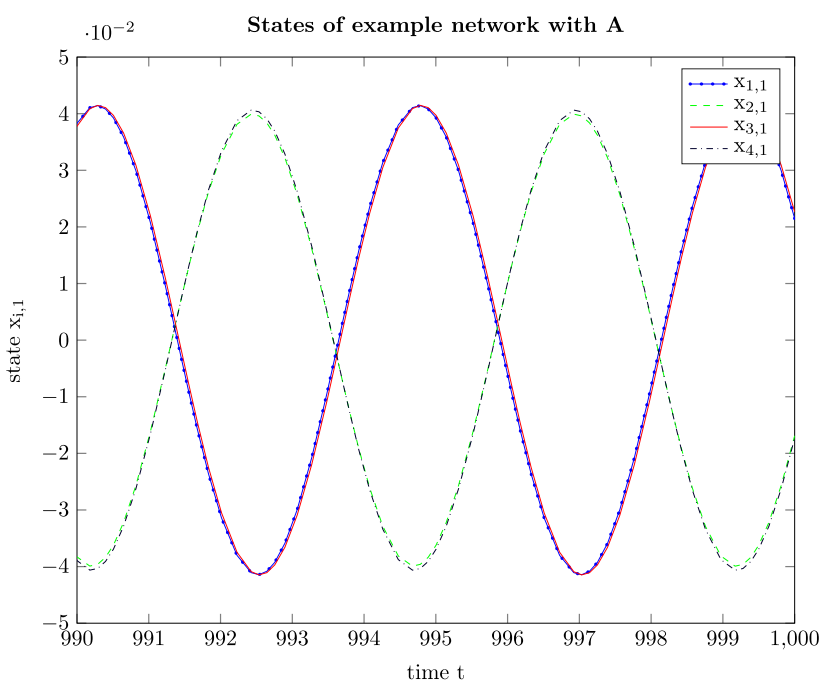

FIG. 9. The states $x_{i, 1}$ of the network with $k=0.3, \tau=2$ in the time interval $[990,1000]$.

below

$$
A=A_{n}+k_{\Delta} \cdot \Delta A,
$$

with $A_{n}, \Delta_{A}$ defined in (38), and $k_{\Delta}=0,0.05,0.10, \ldots, 0.5$.

Synchronization errors of the example network
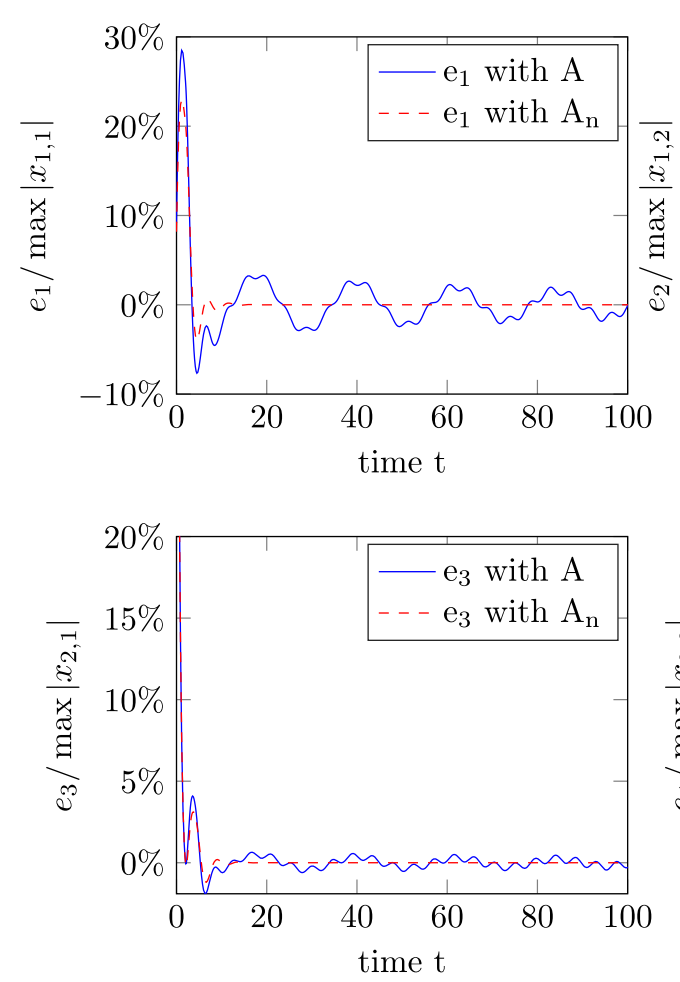
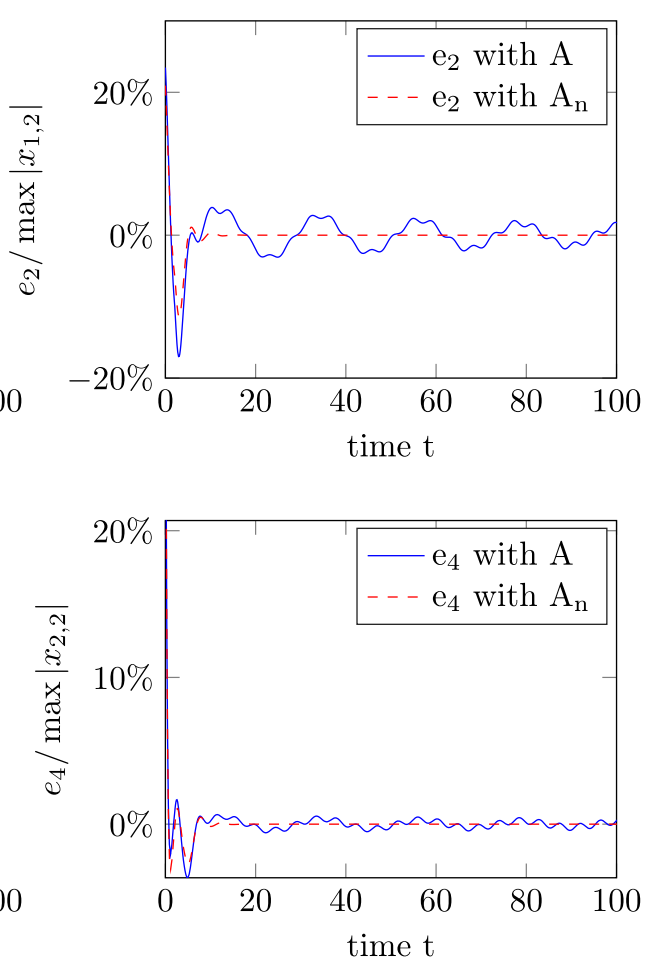

FIG. 10. Synchronization errors of the network with $A$ (practical synchronization, blue colored) and $A_{n}$ (exact synchronization, red colored). 
Synchronization errors of the example network
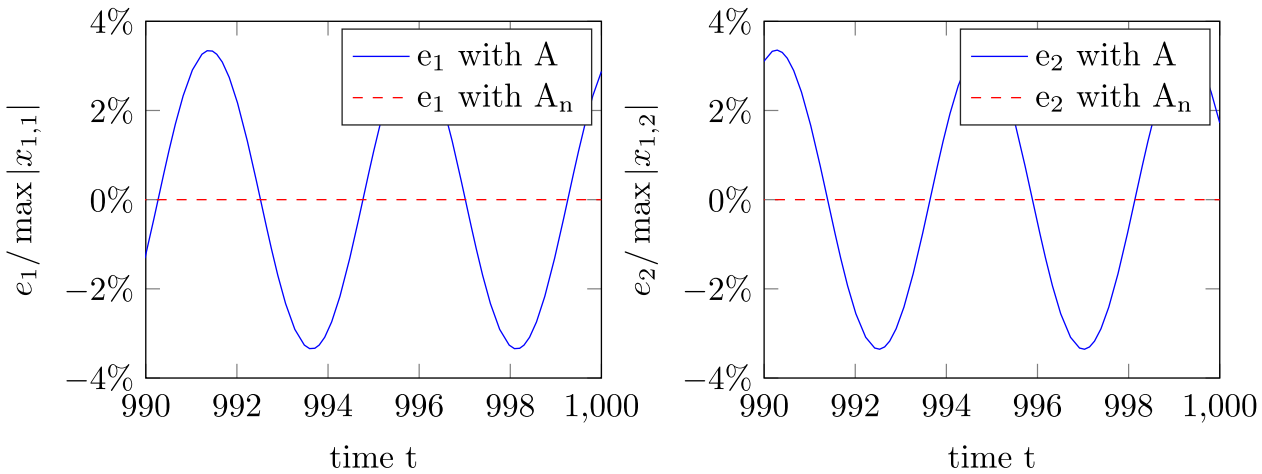

FIG. 11. Synchronization errors of the network with $A$ (practical synchronization, blue colored) and $A_{n}$ (exact synchroniza-
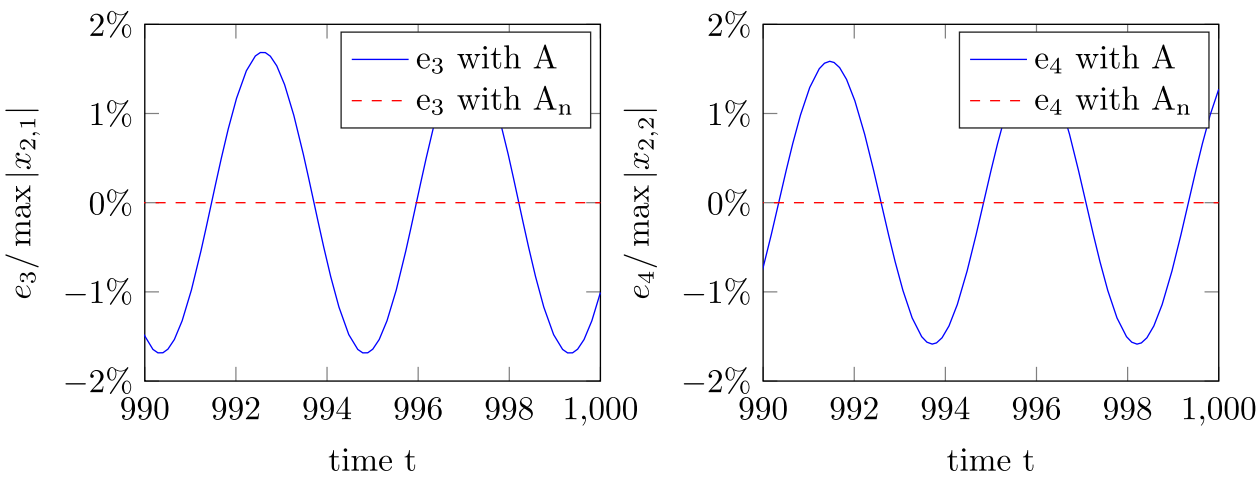
tion, red colored)

The relation between $\left\|e_{i}\right\|$ and $\delta=\left\|k_{\Delta} \Delta A\right\|_{\infty}$ obtained from these simulations is shown in Fig. 12. Here, the signals $x_{1,1}, x_{2,1}$, $x_{1,2}, x_{2,2}, x_{3,1}$, and $x_{4,1}$ are sampled in the time interval $[990,1000]$ to compute the synchronization errors $e_{i}$. Figure 12 shows that the

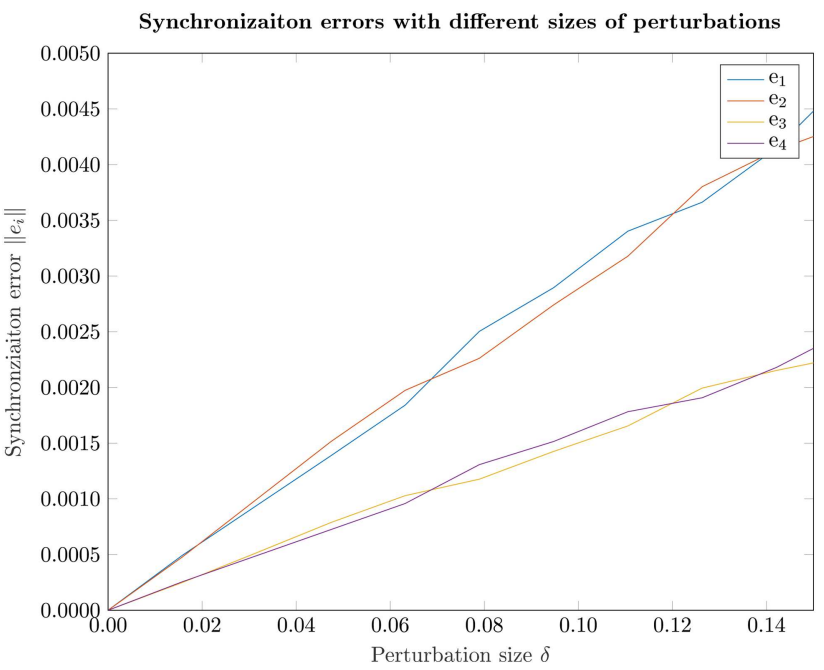

FIG. 12. Synchronization errors with different sizes of perturbations. synchronization error grows with the size of perturbation, and it converges to zero when the perturbation goes to zero.

\section{CONCLUSIONS}

In this paper, practical partial synchronization of delay-coupled systems is studied. First, the synchronization error dynamics are separated from the whole network dynamics for the case where there are perturbations on the coupling, which render exact synchronization impossible. Second, the notion of practical partial synchronization is introduced and sufficient conditions, formulated in terms of LMIs, are provided. The relation between the bounds on the synchronization errors and those of the perturbations is made explicit. From a methodological point of view, a main challenge in the analysis is that the zero solution is no longer an equilibrium point of the synchronization error system. Finally, a numerical example is presented, where the conditions for a network of nonlinear systems to be practically partially synchronized are derived by using one of the theorem proposed in this paper.

The study of practical partial synchronization in this paper is under the assumption of perturbations of the adjacency matrix. It is worth noting that this methodology can also be applied to other situations where the bounded perturbations or uncertainties are present in other components of the network that lead to a violation of the existence conditions for the partial synchronization manifold under consideration (for instance, the vector fields $f_{i}$, input matrices $B_{i}$, output matrices $C_{i}$ ). 


\section{ACKNOWLEDGMENTS}

This work was supported by Project No. C14/17/072 of the KU Leuven Research Council, by Project No. G0A5317N of the Research Foundation-Flanders (FWO-Vlaanderen), and by Project UCoCoS funded by the European Unions Horizon 2020 Research and Innovation Programme under the Marie Sklodowska-Curie Grant Agreement No. 675080.

\section{APPENDIX A: SYNCHRONIZATION ERROR DYNAMICS}

Function $F$ and matrices in (15) are specified as follows:

$$
F\left(\mathscr{X}(t), \mathscr{X}_{r}(t)\right)=\left[\begin{array}{c}
\tilde{f}_{1}\left(x_{1,1}(t)+e_{1,2}(t)\right)-\tilde{f}_{1}\left(x_{1,1}(t)\right) \\
\vdots \\
\tilde{f}_{1}\left(x_{1,1}(t)+e_{1, \kappa_{1}}(t)\right)-\tilde{f}_{1}\left(x_{1,1}(t)\right) \\
\vdots \\
\tilde{f}_{\kappa}\left(x_{\kappa, 1}(t)+e_{\kappa, 2}(t)\right)-\tilde{f}_{\kappa}\left(x_{\kappa, 1}(t)\right) \\
\vdots \\
\tilde{f}_{\kappa}\left(x_{\kappa, 1}(t)+e_{\kappa, \kappa_{\kappa}}(t)\right)-\tilde{f}_{\kappa}\left(x_{\kappa, 1}(t)\right)
\end{array}\right],
$$

$$
A_{1}=\tilde{\mathscr{B}}\left(A_{\text {red }} \otimes I_{m}\right) \tilde{\mathscr{C}},
$$

with

$$
\begin{aligned}
& \tilde{\mathscr{B}}=\operatorname{diag}\left(I_{\kappa_{1}-1} \otimes \tilde{B}_{1}, \ldots, I_{\kappa_{\kappa}-1} \otimes \tilde{B}_{\kappa}\right), \\
& \tilde{\mathscr{C}}=\operatorname{diag}\left(I_{\kappa_{1}-1} \otimes \tilde{C}_{1}, \ldots, I_{\kappa_{\kappa}-1} \otimes \tilde{C}_{\kappa}\right),
\end{aligned}
$$

and

$$
A_{\text {red }}=T_{1} A T_{1}^{T}-T_{2} A T_{1}^{T},
$$

where $T_{1}, T_{2} \in \mathbb{R}^{(N-\kappa) \times N}$ are defined as

with

$T_{1 i}=\left[\begin{array}{ccccc}0 & 1 & \cdots & & 0 \\ \vdots & & \ddots & & \vdots \\ & & & 1 & 0 \\ 0 & & \cdots & 0 & 1\end{array}\right], \quad T_{2 i}=\left[\begin{array}{cccc}1 & 0 & \cdots & 0 \\ 1 & 0 & \cdots & 0 \\ \vdots & \vdots & & \vdots \\ 1 & 0 & \cdots & 0\end{array}\right] \in \mathbb{R}^{\left(\kappa_{i}-1\right) \times \kappa_{i}}$.

For more information on the derivation and a detailed interpretation of $T_{1} A T_{1}^{\top}$ and $T_{2} A T_{1}^{\top}$, refer to Su et al. (2018).

When taking into account perturbations on the adjacency matrix, the additional terms in (18) are described by

$$
A_{2}(\Delta)=k\left[\begin{array}{c}
\operatorname{diag}\left(\sum_{l=1}^{\kappa} \Delta_{1 l, 2}, \ldots, \sum_{l=1}^{\kappa} \Delta_{1 l, \kappa_{1}}\right) \otimes \tilde{B}_{1} \tilde{C}_{1} \\
\ddots \\
0 \quad \operatorname{diag}\left(\sum_{l=1}^{\kappa} \Delta_{1 l, 2}, \ldots, \sum_{l=1}^{\kappa} \Delta_{l l, \kappa_{\kappa}}\right) \otimes \tilde{B}_{\kappa} \tilde{C}_{\kappa}
\end{array}\right],
$$

where

$$
A_{\Delta}=\left[\begin{array}{ccc}
\operatorname{diag}\left(\Delta_{11,2}, \ldots, \Delta_{11, \kappa_{1}}\right) & \cdots & \operatorname{diag}\left(\Delta_{1 \kappa, 2}, \ldots, \Delta_{1 \kappa, \kappa_{1}}\right) \\
\vdots & \ddots & \vdots \\
\operatorname{diag}\left(\Delta_{\kappa 1,2}, \ldots, \Delta_{\kappa 1, \kappa_{\kappa}}\right) & \cdots & \operatorname{diag}\left(\Delta_{\kappa \kappa, 2}, \ldots, \Delta_{\kappa \kappa, \kappa_{\kappa}}\right)
\end{array}\right] .
$$

\section{APPENDIX B: PROOF OF THEOREM 1}

Proof. Considering a Lyapunov-Krasovskii functional

$$
v\left(\mathscr{X}_{t}\right)=\mathscr{X}^{\top}(t) P \mathscr{X}(t)+\int_{-\tau}^{0} \mathscr{X}^{\top}(t+\theta) e^{2 \sigma \theta} \mathrm{Q} \mathscr{X}(t+\theta) \mathrm{d} \theta,
$$

we have

$$
\alpha_{1}\|\mathscr{X}(t)\|^{2} \leq v\left(\mathscr{X}_{t}\right) \leq \alpha_{2}\left\|\mathscr{X}_{t}\right\|_{s}^{2}
$$

where $\alpha_{1}=\lambda_{\min }(P)$ and $\alpha_{2}=\lambda_{\max }(P)+\tau \lambda_{\max }(Q)$.
Then,

$$
\begin{aligned}
& \frac{d}{d t} v\left(\mathscr{X}_{t}\right)=2 \mathscr{X}^{\top}(t) P\left[F\left(\mathscr{X}(t), \mathscr{X}_{r}(t)\right)-k A_{0} \mathscr{X}(t)\right. \\
&\left.+k A_{1} \mathscr{X}(t-\tau)+n(t, \tau, \Delta)\right] \\
&+\mathscr{X}^{\top}(t) Q \mathscr{X}(t)-\mathscr{X}^{\top}(t-\tau) e^{-2 \sigma \tau} \mathrm{Q} \mathscr{X}(t-\tau) \\
&-2 \sigma \int_{-\tau}^{0} \mathscr{X}^{\top}(t+\theta) e^{-2 \sigma \theta} \mathrm{Q} \mathscr{X}(t+\theta) d \theta
\end{aligned}
$$

Here, $2 \mathscr{X}^{\top}(t) P n(t, \tau, \Delta) \leq 2\|\mathscr{X}(t)\|\|P\|\|n\|$, which implies

$$
2 \mathscr{X}^{\top}(t) P n(t) \leq 2 \frac{\gamma\|P\|}{\sqrt{\alpha_{1}}} \sqrt{v\left(\mathscr{X}_{t}\right)} .
$$

The other term

$2 \mathscr{X}^{\top}(t) P F\left(\mathscr{X}(t), \mathscr{X}_{r}(t)\right) \leq 2\left\|\mathscr{X}^{\top}(t)\right\|\|P\| L\|\mathscr{X}(t)\| \leq 2 \frac{v\left(\mathscr{X}_{t}\right)}{\alpha_{1}}\|P\| L$. 
Then, we arrive at

$$
\begin{aligned}
\frac{d}{d t} v\left(\mathscr{X}_{t}\right) \leq & {\left[\begin{array}{c}
\mathscr{X}(t) \\
\mathscr{X}(t-\tau)
\end{array}\right]^{\top} \mathscr{M}(P, Q)\left[\begin{array}{c}
\mathscr{X}(t) \\
\mathscr{X}(t-\tau)
\end{array}\right] } \\
& -2 \sigma \int_{-\tau}^{0} \mathscr{X}^{\top}(t+\theta) e^{2 \sigma \theta} Q \mathscr{X}(t+\theta) d \theta \\
& +2 \frac{\gamma\|P\|}{\sqrt{\alpha_{1}}} \sqrt{v\left(\mathscr{X}_{t}\right)}+2 \frac{\|P\| L}{\alpha_{1}} v\left(\mathscr{X}_{t}\right) .
\end{aligned}
$$

Note that the functional can be rewritten as

$$
\begin{aligned}
v\left(\mathscr{X}_{t}\right)= & {\left[\begin{array}{c}
\mathscr{X}(t) \\
\mathscr{X}(t-\tau)
\end{array}\right]^{\top}\left[\begin{array}{ll}
P & 0 \\
0 & 0
\end{array}\right]\left[\begin{array}{c}
\mathscr{X}(t) \\
\mathscr{X}(t-\tau)
\end{array}\right] } \\
& +\int_{-\tau}^{0} \mathscr{X}^{\top}(t+\theta) e^{2 \sigma \theta} \mathrm{Q} \mathscr{X}(t+\theta) d \theta .
\end{aligned}
$$

So, we can derive

$$
\begin{aligned}
& \frac{d}{d t} v\left(\mathscr{X}_{t}\right)+2\left(\sigma-\frac{L\|P\|}{\alpha_{1}}\right) v\left(\mathscr{X}_{t}\right)-2 \frac{\gamma\|P\|}{\sqrt{\alpha_{1}}} \sqrt{v\left(\mathscr{X}_{t}\right)} \\
& \quad \leq\left[\begin{array}{c}
\mathscr{X}(t) \\
\mathscr{X}(t-\tau)
\end{array}\right]^{\top}(\mathscr{M}(P, Q)+2 \sigma \mathscr{N}(P))\left[\begin{array}{c}
\mathscr{X}(t) \\
\mathscr{X}(t-\tau)
\end{array}\right],
\end{aligned}
$$

with $\mathscr{M}(P, Q)$ and $\mathscr{N}(P)$ defined in Theorem 1 .

We can conclude from the above arguments that if condition (7) holds, then

$$
\frac{d}{d t} v\left(\mathscr{X}_{t}\right) \leq-2\left(\sigma-\frac{L\|P\|}{\alpha_{1}}\right) v\left(\mathscr{X}_{t}\right)+2 \frac{\gamma\|P\|}{\sqrt{\alpha_{1}}} \sqrt{v\left(\mathscr{X}_{t}\right)}
$$

If $L<\frac{\alpha_{1}}{\|P\|} \sigma=\frac{\lambda_{\min }(P)}{\lambda_{\max }(P)} \sigma$, it can be shown that

$$
\begin{aligned}
\left\|\mathscr{X}_{t}\right\| \leq & \frac{\sqrt{\lambda_{\max }(P)+\tau \lambda_{\max }(Q)}}{\sqrt{\lambda_{\min }(P)}} e^{-\left(\sigma-\frac{L \lambda_{\max }(P)}{\lambda_{\min }(P)}\right) t}\|\Psi\|_{s} \\
& +\frac{\gamma \lambda_{\max }(P)}{\sigma \lambda_{\min }(P)-L \lambda_{\max }(P)}\left(1-e^{-\left(\sigma-\frac{L \lambda_{\max }(P)}{\lambda_{\min }(P)}\right) t}\right)
\end{aligned}
$$

It follows that if $L<\frac{\lambda_{\min }(P)}{\lambda_{\max }(P)} \sigma$, system (18) is practically stable with $\mu$ and $T$ chosen as below

$$
\begin{aligned}
& \text { for }\|\Psi\|_{s} \leq \frac{\gamma \lambda_{\max }(P)}{\left(\sigma-\frac{L \lambda_{\max }(P)}{\lambda_{\min }(P)}\right) \sqrt{\lambda_{\min }(P)\left[\lambda_{\max }(P)+\tau \lambda_{\max }(Q)\right]}}, \\
& \mu=\beta(\gamma)=\frac{\gamma \lambda_{\max }(P)}{\sigma \lambda_{\min }(P)-L \lambda_{\max }(P)}, \\
& T=0,
\end{aligned}
$$

$$
\begin{aligned}
& \mu=\beta(\gamma)=\frac{k_{\mu} \gamma \lambda_{\max }(P)}{\sigma \lambda_{\min }(P)-L \lambda_{\max }(P)}, \\
& T=\frac{1}{\sigma-\frac{L \lambda_{\max }(P)}{\lambda_{\min }(P)}} \ln \left(\frac{\left(\sigma-\frac{L \lambda_{\max }(P)}{\lambda_{\min }(P)}\right) \sqrt{\lambda_{\min }(P)\left[\lambda_{\max }(P)+\tau \lambda_{\max }(Q)\right]}\|\Psi\|_{s}-\gamma \lambda_{\max }(P)}{\left(\sigma \lambda_{\min }(P)-L \lambda_{\max }(P)\right) \mu-\gamma \lambda_{\max }(P)}\right),
\end{aligned}
$$

where $k_{\mu}$ satisfies the condition $1<k_{\mu}<$ $\frac{\left(\sigma-\frac{L \lambda_{\max }(P)}{\lambda_{\min }(P)}\right) \sqrt{\lambda_{\min }(P)\left[\lambda_{\max }(P)+\tau \lambda_{\max }(Q)\right]}\|\Psi\|_{s}}{\gamma \lambda_{\max }(P)}$, and $\|\Psi\|_{s}$ is the supremum $\operatorname{norm}\|\Psi(\theta)\|_{s}:=\sup _{\theta \in[0-\tau]}^{\gamma \lambda_{\max }(P)}\|\Psi(\theta)\|$.

\section{APPENDIX C: PROOF OF THEOREM 2}

Proof. Consider a Lyapunov-Krasovskii functional

$$
\begin{aligned}
v\left(\mathscr{X}_{t}\right)= & \mathscr{X}^{\top}(t) P \mathscr{X}(t)+\int_{t-\tau}^{t} \mathscr{X}^{\top}(\theta) e^{2 \sigma(\theta-t)} \mathrm{Q} \mathscr{X}(\theta) \mathrm{d} \theta \\
& +\tau \int_{-\tau}^{0} \int_{t+s}^{s} \dot{X}^{\top}(\theta) e^{2 \sigma(\theta-t)} Z \dot{X}(\theta) \mathrm{d} \theta \mathrm{d} s .
\end{aligned}
$$

In fact, this Lyapunov-Krasovskii functional is defined in $\mathscr{C}([-2 \tau, 0]$, $\left.\mathbb{R}^{N n-\kappa n}\right)$, which is a subspace of $\mathscr{C}\left([-\tau, 0], \mathbb{R}^{N n-\kappa n}\right)$. Hence, the conditions derived from this functional is still sufficient for the stability problem on $[-\tau, 0]$.
Obviously, we have

$$
\alpha_{1}\|\mathscr{X}(t)\|^{2} \leq v\left(\mathscr{X}_{t}\right) \leq \alpha_{2}\left\|\mathscr{X}_{t}\right\|_{s}^{2}
$$

where $\alpha_{1}=\lambda_{\min }(P)$ and $\alpha_{2}=\lambda_{\max }(P)+\tau \lambda_{\max }(Q)+\tau \lambda_{\max }(Z)$, and we can derive

$$
\begin{aligned}
\dot{v}\left(\mathscr{X}_{t}\right) \leq & 2 \mathscr{X}^{\top}(t) P\left(F\left(\mathscr{X}(t), \mathscr{X}_{r}(t)\right)\right. \\
& \left.-k A_{0} \mathscr{X}(t)+k A_{1} \mathscr{X}(t-\tau)+n(t, \tau, \Delta)\right) \\
& +\mathscr{X}^{\top}(t) Q \mathscr{X}(t)-e^{-2 \sigma \tau} \mathscr{X}^{\top}(t-\tau) Q \mathscr{X}(t-\tau) \\
& -2 \sigma \int_{t-\tau}^{t} \mathscr{X}^{\top}(\theta) e^{2 \sigma(\theta-t)} Q \mathscr{X}(\theta) \mathrm{d} \theta \\
& +\tau^{2} \dot{\mathscr{X}}^{\top}(t) Q \dot{\mathscr{X}}(t)-\tau e^{-2 \sigma \tau} \int_{t-\tau}^{\tau} \dot{\mathscr{X}}^{\top}(\theta) Z \dot{\mathscr{X}}(\theta) \mathrm{d} \theta \\
& -2 \sigma \tau \int_{-\tau}^{0} \int_{t+s}^{s} \dot{\mathscr{X}}^{\top}(\theta) e^{2 \sigma(\theta-t)} Z \dot{\mathscr{X}}(\theta) \mathrm{d} \theta \mathrm{d} s .
\end{aligned}
$$


Using Jensen's inequality, it follows that

$$
\begin{aligned}
\dot{v}\left(\mathscr{X}_{t}\right) \leq & 2 \mathscr{X}^{\top}(t) P\left(F\left(\mathscr{X}(t), \mathscr{X}_{r}(t)\right)-k A_{0} \mathscr{X}(t)+k A_{1} \mathscr{X}(t-\tau)\right. \\
& +n(t, \tau, \Delta))+2 \sigma \mathscr{X}^{\top}(t) P \mathscr{X}(t)+\mathscr{X}^{\top}(t) Q \mathscr{X}(t) \\
& -e^{-2 \sigma \tau} \mathscr{X}^{\top}(t-\tau) Q \mathscr{X}(t-\tau)+\tau^{2} \dot{\mathscr{X}}^{\top}(t) Q \mathscr{X}(t) \\
& -e^{-2 \sigma \tau}(\mathscr{X}(t)-\mathscr{X}(t-\tau))^{\top} Z(\mathscr{X}(t)-\mathscr{X}(t-\tau)) \\
& -2 \sigma v\left(\mathscr{X}_{t}\right) .
\end{aligned}
$$

With the inequalities (31) in mind, we have for any diagonal matrix $V>0$

$$
\left[\begin{array}{c}
\mathscr{X} \\
F\left(\mathscr{X}(t), \mathscr{X}_{r}(t)\right)
\end{array}\right]^{\top}\left[\begin{array}{cc}
F_{1} V & -F_{2} V \\
* & V
\end{array}\right]\left[\begin{array}{c}
\mathscr{X} \\
F\left(\mathscr{X}(t), \mathscr{X}_{r}(t)\right)
\end{array}\right] \leq 0 .
$$

On the other hand, we have from (32) that

$$
\begin{aligned}
& {\left[\begin{array}{c}
\mathscr{X}(t) \\
\mathscr{X}(t-\tau) \\
F\left(\mathscr{X}(t), \mathscr{X}_{r}(t)\right) \\
n(t, \tau, \Delta)
\end{array}\right]^{\top}\left[\Theta+H_{1} P H_{2}+H_{2}^{\top} P H_{1}^{\top}+\tau^{2} H_{2}^{\top} Z H_{2}\right]} \\
& \quad \times\left[\begin{array}{c}
\mathscr{X}(t) \\
\mathscr{X}(t-\tau) \\
F\left(\mathscr{X}(t), \mathscr{X}_{r}(t)\right) \\
n(t, \tau, \Delta)
\end{array}\right]<0 .
\end{aligned}
$$

Combining (C) with (C3), we can conclude

$$
\dot{v}\left(\mathscr{X}_{t}\right)<-2 \sigma v\left(\mathscr{X}_{t}\right)+n(t, \tau, \Delta)^{\top} W n(t, \tau, \Delta) .
$$

Hence,

$$
\dot{v}\left(\mathscr{X}_{t}\right)<-2 \sigma v\left(\mathscr{X}_{t}\right)+\lambda_{\max }(W) \gamma^{2} .
$$

Using Grönwall's inequality, we arrive at

$$
v\left(\mathscr{X}_{t}\right)<v(\Psi) e^{-2 \sigma t}-\frac{\gamma^{2}}{2 \sigma} \lambda_{\max }(W)\left(e^{-2 \sigma t}-1\right) .
$$

Now, it follows from (C2) that

$$
\left\|\mathscr{X}_{t}\right\| \leq \sqrt{\frac{\gamma^{2}}{2 \sigma \alpha_{1}} \lambda_{\max }(W)+e^{-2 \sigma t}\left(\frac{\alpha_{2}}{\alpha_{1}}\|\Psi\|_{s}^{2}-\frac{\gamma^{2}}{2 \sigma \alpha_{1}} \lambda_{\max }(W)\right)} .
$$

Observe that for an initial condition $\Psi$ such that $\|\Psi\|_{s} \leq \gamma \sqrt{\frac{\lambda_{\max }(W)}{2 \sigma \alpha_{2}}}$, we have

$$
\left\|\mathscr{X}_{t}\right\| \leq \mu=\gamma \sqrt{\frac{\lambda_{\max }(W)}{2 \sigma \alpha_{1}}}, \quad \forall t \geq 0 .
$$

For an initial condition $\Psi$ such that $\|\Psi\|_{s}>\gamma \sqrt{\frac{\lambda_{\max }(W)}{2 \sigma \alpha_{2}}}$, we have

$$
\left\|\mathscr{X}_{t}\right\| \leq \mu, \quad \forall t \geq T(\mu, \Psi),
$$

where $\mu=k_{\mu} \gamma \sqrt{\frac{\lambda_{\max }(W)}{2 \sigma \alpha_{1}}}, 1<k_{\mu}<\frac{1}{\gamma} \sqrt{\frac{2 \sigma \alpha_{2}}{\lambda_{\max }(W)}}\|\Psi\|_{s}$, and the time $T(\mu, \Psi)$ is obtained from the condition

$$
0<e^{-2 \sigma t}\left(\frac{\alpha_{2}}{\alpha_{1}}\|\Psi\|^{2}-\frac{\gamma^{2}}{2 \sigma \alpha_{1}} \lambda_{\max }(W)\right)<\mu^{2}-\frac{\gamma^{2}}{2 \sigma \alpha_{1}} \lambda_{\max }(W),
$$

thus,

$$
T=\frac{1}{2 \sigma} \ln \left(\frac{2 \sigma \alpha_{2}\|\Psi\|_{s}^{2}-\gamma^{2} \lambda_{\max }(W)}{2 \sigma \alpha_{1} \mu^{2}-\gamma^{2} \lambda_{\max }(W)}\right)
$$

\section{APPENDIX D: PROOF OF SEMIPASSIVITY OF SYSTEM (1 1 )}

Equation (11) is of the form (1) with

$$
\left\{\begin{array}{l}
\dot{x}_{i}(t)=f\left(x_{i}(t)\right)+B_{i} u_{i}(t) \\
y_{i}(t)=C_{i} x_{i}(t)
\end{array}\right.
$$

where

$$
\begin{array}{ll}
x_{i}=\left[\begin{array}{l}
x_{i, 1} \\
x_{i, 2}
\end{array}\right], \quad y_{i}=\left[\begin{array}{l}
y_{i, 1} \\
y_{i, 2}
\end{array}\right], \quad u_{i}=\left[\begin{array}{l}
u_{i, 1} \\
u_{i, 2}
\end{array}\right], \\
f\left(x_{i}\right)=\left[\begin{array}{l}
-\varepsilon x_{i, 1}+x_{i, 2}-x_{i, 1}\left(x_{i, 1}^{2}+x_{i, 2}^{2}\right) \\
-x_{i, 1}-\varepsilon x_{i, 2}-x_{i, 2}\left(x_{i, 1}^{2}+x_{i, 2}^{2}\right)
\end{array}\right], \quad B_{i}=\left[\begin{array}{ll}
0 & 1 \\
1 & 0
\end{array}\right], \\
C_{i}=\left[\begin{array}{l}
x_{i, 2} \\
x_{i, 1}
\end{array}\right] .
\end{array}
$$

Define the so-called storage function $V\left(x_{i}\right)$ as

$$
V\left(x_{i}\right)=\frac{1}{2}\left(x_{i, 1}^{2}+x_{i, 2}^{2}\right)
$$

and the scalar function $S\left(x_{i}\right)$ as

$$
S\left(x_{i}\right)=2 \varepsilon V\left(x_{i}\right)+4 V^{2}\left(x_{i}\right)
$$

which is positive outside the ball $\mathscr{B}=\left\{x_{i} \in \mathbb{R}^{2} \mid V\left(x_{i}\right) \leq \frac{\max (-\epsilon, 0)}{2}\right\}$. Then, we have

$$
\begin{aligned}
\dot{V}\left(x_{i}\right) & =x_{i, 1} u_{i, 2}+x_{i, 2} u_{i, 1}-\left[\varepsilon\left(x_{i, 1}^{2}+x_{i, 2}^{2}\right)+k_{i}\left(x_{i, 1}^{2}+x_{i, 2}^{2}\right)^{2}\right] \\
& =y^{\top} u-2 \varepsilon V\left(x_{i}\right)-4 V^{2}\left(x_{i}\right) \\
& \leq y^{\top} u-S\left(x_{i}\right) .
\end{aligned}
$$

That is, condition (4) is satisfied.

\section{APPENDIX E: SOLUTION OF LMIS (32) IN EXAMPLE 1 IN SEC. VI}

$P=\left[\begin{array}{cccc}19.6194 & 1.8757 & 4.7948 & 2.5742 \\ 1.8757 & 19.8934 & 0.9150 & 7.3896 \\ 4.7948 & 0.9150 & 17.8032 & 10.0527 \\ 2.5742 & 7.3896 & 10.0527 & 23.7725\end{array}\right]$




$\begin{aligned} Q & =\left[\begin{array}{cccc}11.1995 & 2.3924 & 1.2749 & 0.5651 \\ 2.3924 & 9.2019 & 0.5942 & 3.8496 \\ 1.2749 & 0.5942 & 8.6784 & 4.5230 \\ 0.5651 & 3.8496 & 4.5230 & 15.9833\end{array}\right], \\ Z & =\left[\begin{array}{cccc}0.2907 & 0.0351 & 0.2097 & 0.0774 \\ 0.0351 & 0.3373 & 0.1165 & 0.4194 \\ 0.2097 & 0.1165 & 0.2348 & 0.2100 \\ 0.0774 & 0.4194 & 0.2100 & 0.6446\end{array}\right], \\ V & =\left[\begin{array}{ccccc}350.6328 & 0 & 0 & 0 & \\ 0 & 386.4757 & 0 & 0 \\ 0 & 0 & 16.2167 & 0 \\ 0 & 0 & 0 & 148.6860\end{array}\right], \\ W & =\left[\begin{array}{ccccc}354.5293 & 0.9549 & -135.9724 & -19.9116 \\ 0.9549 & 410.8246 & -24.8248 & -116.4935 \\ -135.9724 & -24.8248 & 391.4282 & 64.0708 \\ -19.9116 & -116.4935 & 64.0708 & 302.1782\end{array}\right] .\end{aligned}$

\section{REFERENCES}

Abrams, D. M. and Strogatz, S. H., "Chimera states for coupled oscillators," Phys. Rev. Lett. 93, 174102 (2004).

Bollobas, B., Modern Graph Theory (Springer-Verlag GmbH, 1998).

Dahms, T., Lehnert, J., and Schöll, E., "Cluster and group synchronization in delaycoupled networks,” Phys. Rev. E 86, 016202 (2012).

DeLellis, P., di Bernardo, M., and Russo, G., "On QUAD, Lipschitz, and contracting vector fields for consensus and synchronization of networks," IEEE Trans. Circuits Syst. I Reg. Pap. 58, 576-583 (2011).

Gibbons, A., Algorithmic Graph Theory (Cambridge University Press, 1985).

Gray, C. M., "Synchronous oscillations in neuronal systems: Mechanisms and functions," J. Comput. Neurosci. 1, 11-38 (1994).

Hagerstrom, A. M., Murphy, T. E., Roy, R., Hövel, P., Omelchenko, I., and Schöll, E., "Experimental observation of chimeras in coupled-map lattices," Nat. Phys. 8, 658-661 (2012).

Khalil, H. K., Nonlinear Systems (PEARSON-SUPERPEDIDO, 2001).

Kuramoto, Y., "Chemical waves," in Chemical Oscillations, Waves, and Turbulence (Springer, Berlin, 1984), pp. 89-110.

Löfberg, J., "YALMIP : A toolbox for modeling and optimization in MATLAB," in 2004 IEEE International Conference on Robotics and Automation (IEEE Cat. No.04CH37508) (IEEE, 2004), pp. 284-289.
Martens, E. A., Thutupalli, S., Fourriere, A., and Hallatschek, O., "Chimera states in mechanical oscillator networks," Proc. Natl. Acad. Sci. U.S.A. 110, 10563-10567 (2013).

Montenbruck, J. M., Bürger, M., and Allgöwer, F., "Practical synchronization with diffusive couplings," Automatica 53, 235-243 (2015).

Orosz, G., "Decomposition of nonlinear delayed networks around cluster states with applications to neurodynamics," SIAM J. Appl. Dyn. Syst. 13, 1353-1386 (2014).

Panteley, E. and Loria, A., "Synchronization and dynamic consensus of heterogeneous networked systems," IEEE Trans. Automat. Contr. 62, 3758-3773 (2017).

Pogromsky, A. Y., “Passivity based design of synchronizing systems," Int. J. Bifurcat. Chaos 08, 295-319 (1998).

Ryono, K. and Oguchi, T., "Partial synchronization in networks of nonlinear systems with transmission delay couplings," IFAC-PapersOnLine 48, 77-82 (2015).

Sawicki, J., Omelchenko, I., Zakharova, A., and Schöll, E., "Delay-induced chimeras in neural networks with fractal topology," Eur. Phys. J. B 92, 54 (2019).

Schmidt, L., Schönleber, K., Krischer, K., and García-Morales, V., "Coexistence of synchrony and incoherence in oscillatory media under nonlinear global coupling," Chaos 24, 013102 (2014).

Steur, E. and Nijmeijer, H., "Synchronization in networks of diffusively time-delay coupled (semi-)passive systems," IEEE Trans. Circuits Syst. I Reg. Pap. 58, 1358-1371 (2011)

Steur, E., Oguchi, T., van Leeuwen, C., and Nijmeijer, H., "Partial synchronization in diffusively time-delay coupled oscillator networks," Chaos 22, 043144 (2012).

Steur, E., Ünal, H. U., van Leeuwen, C., and Michiels, W., "Characterization and computation of partial synchronization manifolds for diffusive delay-coupled systems," SIAM J. Appl. Dyn. Syst. 15, 1874-1915 (2016).

Steur, E., Vromen, T., and Nijmeijer, H., Practical Synchronization in Networks of Diffusively Coupled Non-Identical Systems (Eindhoven University of Technology, 2015), Vol. 2015.031, D\&C

Su, L., Michiels, W., Steur, E., and Nijmeijer, H., "A method for computation and analysis of partial synchronization manifolds of delay coupled systems," in Advances in Delays and Dynamics (Springer Verlag, 2018).

Tinsley, M. R., Nkomo, S., and Showalter, K., "Chimera and phase-cluster states in populations of coupled chemical oscillators," Nat. Phys. 8, 662-665 (2012).

Toh, K. C., Todd, M. J., and Tütüncü, R. H., "SDPT3-A MATLAB software package for semidefinite programming," Optim. Methods Softw. 11, 545-581 (1999).

Villafuerte, R., Mondié, S., and Poznyak, A., "Practical stability of time-delay systems: LMI's approach,” Eur. J. Control 17, 127-138 (2011).

Vromen, T. G. M., Steur, E., and Nijmeijer, H., "Training a network of electronic neurons for control of a mobile robot," Int. J. Bifurcat. Chaos 26, 1650196 (2016). 